\title{
Outage and Error Analysis of Dual-Hop TAS/MRC MIMO RF-UOWC Systems
}

\author{
Imran Shafique Ansari (i), Member, IEEE, Latif Jan, Member, IEEE, Yutong Tang (®), Liang Yang (i), Member, IEEE, \\ and Mohammad Haseeb Zafar ${ }^{(0)}$, Senior Member, IEEE
}

\begin{abstract}
This paper gives an insight on the performance of mixed dual-hop radio-frequency (RF)-underwater optical wireless communication (UOWC) systems. The system consists of multipleinput multiple-output (MIMO) RF hop employing Nakagami-m fading channel on the source $(S)$ node communicating with a destination node $(D)$ considered as the legitimate receiver via an amplify-and-forward (AF) relay $(R)$ node equipped with multiple RF antennas for reception. It considers transmit antenna selection (TAS) scheme for communication in the MIMO RF hop while the information is transmitted from the $S$ node to the $D$ node, i.e. submarine etc., via the UOWC hop. Specifically, the $R$ node receives incoming information messages from $S$ node via MIMO RF links, applies maximal-ratio combining (MRC) technique, amplifies the output combined signal, and subsequently forwards it to the destination utilising a variable gain relaying (VGR) via an UOWC link. We derive exact closed-form expressions for the system's end-toend (E2E) statistical channel characteristics. Our derived analytical expressions present an efficient technique to depict the impact of our system and channel parameters on the performance, namely the varying number of increasing antennas $N_{t}=N_{r}=2,3,4$ or more from the $S$ node towards $R$ node and the involvement of underwater detection techniques of $r=1$ for heterodyne detection and $r=2$ for intensity modulation/direct detection (IM/DD) in the underwater turbulence severity of the UOWC link. Outage probability (OP) and average bit error rate (BER) closed-form expressions for the varying bubble levels $(\mathrm{BL})(\mathrm{L} / \mathrm{min})$ for different scenarios, varying temperature gradients (TG) $\left({ }^{\circ} \mathrm{C} \mathrm{cm}^{-1}\right)$, different fresh and saline waters, and various binary modulation techniques have been accurately validated for the E2E system presented in this work along with the tightness of their respective high-end asymptotes.
\end{abstract}

Index Terms-Radio-frequency (RF), underwater optical wireless communication (UOWC), dual-hop relaying, multipleinput multiple-output (MIMO), amplify-and-forward, variable

Manuscript received May 23, 2021; revised July 27, 2021; accepted August 1, 2021. Date of publication August 5, 2021; date of current version October 15,2021 . This work was supported in part by the Engineering and Physical Sciences Research Council (EPSRC) under Grant EP/R041660/1: Bandwidth and Energy Efficient Compact Multi-Antenna Systems for Connected Autonomous Vehicles. The review of this article was coordinated by Prof. Wan Choi. (Corresponding author: Imran Shafique Ansari.)

Imran Shafique Ansari is with the James Watt School of Engineering, University of Glasgow, Glasgow G12 8QQ, U.K. (e-mail: ansarimrans@ gmail.com).

Latif Jan and Mohammad Haseeb Zafar are with the Department of Electrical Engineering, University of Engineering and Technology, Peshawar 25120, Pakistan (e-mail: latifjn86@gmail.com; haseeb@uetpeshawar.edu.pk).

Yutong Tang is with the University of Electronic Science and Technology of China, Chengdu 610054, China (e-mail: yutongtang19981118@outlook.com).

Liang Yang is with the College of Computer Science and Electronic Engineering, Hunan University, Changsha 410082, China, and also with the State key Laboratory of Networking and Switching Technology, Beijing University of Posts and Telecommunications, Beijing 100876, China (e-mail: liangy@ hnu.edu.cn).

Digital Object Identifier 10.1109/TVT.2021.3102837 gain, maximal-ratio combining (MRC), outage probability, and bit error rate (BER).

\section{INTRODUCTION}

\section{A. Background}

$\mathbf{U}$ NDERWATER optical wireless communications (UOWC) is gaining an eminence attention via combining its mixed communication strategies with radio-frequency (RF) and beyond. It is providing the most awaited surprises and several new technological advances, especially from spectral efficiency perspective, thereby paving its path to be one of the potential contender technologies towards the next generation of cellular networks in [1]. The importance and effectiveness due to the real-time and ultra-high applications of UOWC are mentioned in [2] while the acoustic counterpart to form the so-called acoustic-optical hybrid communications are described in [3]. Nevertheless, the performance of UOWC systems is primarily limited by absorption, scattering, and turbulence. Absorption and scattering that depict the energy loss and direction deviation when photons propagate underwater, have been well studied in [4]. Turbulence is defined as the rapid fluctuation of the refractive index along the path of the optical field traversing the water medium, caused by the variations in temperature, pressure, salinity, and air bubbles, resulting in signal fading that impairs the UOWC system performance. On the other hand, typical link configurations of UOWC systems include horizontal and vertical configurations. While horizontal configuration has been well studied so far, vertical one has not received enough attention apart from some coverage in [5], [6] till date.

UOWC is one of the most promising and most emerged phenomena in this era of technology [7] while the most severely affected optical characteristics in underwater are caused by the absorption, scattering, and turbulence's in [8]. Though absorption and scattering are the other two impairments in underwater as discussed above, the losses incurred due to turbulence's are enormous and cannot be neglected in this discussion as explained in [8], [9].

Water's refractive index changes rapidly due to turbulence's, considering the bubble levels, temperature and pressure inhomogeneities in the sea channel studied in [10]. It can be affected abruptly due to water pressure, temperature, and most specially the ocean currents. Likewise, the analysis on the effect of salinity 
is evaluated in [11] and the modulation techniques of an UOWC are equally important factors for consideration in this work reflected in [12].

On the other hand, wide deployment of multiple antennas termed as multiple-input multiple-output (MIMO) in wireless relay networks has been recognised prominently in battling the increase in transmission reliability and the fading strategies in the channel [13]. Pivotal studies and its deployment in the use of amplify-and-forward (AF) relaying technology is considered beneficial for multiple antennas and correspondingly its capability of enabling beam-forming strategies are discussed in [14]. Moreover, an increase in the number of antennas on both ends is a continuous system design challenge thereby rendering its deployment quite complex [15]. Additionally, the signal that is processed by the MIMO transceivers increases exponentially with the increase in the number of antennas [16]. Inspired by the efficient strategy of the antenna selection that is more useful to improve the implementation complexity, it manipulates the diversity of the transmit and receive antennas. Furthermore, antenna selection reduces the signal processing complexity and its feedback overhead. In [17], the dual-hop MIMO relaying with antenna selection performance in Rayleigh fading was evaluated by giving an insight on the probability density function (PDF) for the instantaneous end-to-end (E2E) signal-to-noise ratio (SNR).

\section{B. Related Literature}

The scientific research community continues to show a keen interest in the direction of UOWC as demonstrated via incredible experimental work in [10], [12], [18], with keen involvement in the use of RF and underwater technologies especially since 2018 on-wards till [19], and presenting some thorough performance analysis work in [1], [2]. Quite importantly, underwater technologies pose to become dominant in the 21 st century as it continues to highlight its importance in the comprehension of many potential applications, e.g., military surveillance, oil extractions and production, ecological monitoring, and foremost important the climate-based activities in [20]. Due to many constraints in terms of scarce and limited bandwidth, neither the traditional RF nor the acoustic technologies provide the most efficient high-speed underwater communication [21]. Additionally, the acoustic links mostly experience time delays in their communication systems as recently highlighted in [22]. As a result, its operation in the real-time speed for underwater communications through acoustic medium poses a challenge till-date due to its impairments in [21] in the presence of scattering, absorption, and turbulence's.

In contrast to acoustic underwater communications technology, UOWC technology has gained enormous consideration as a key-enabling technology for larger capacity in high-speed underwater communications [7], [9], [23]. In comparison to the acoustic communication, UOWC technology offers a data-rate up to tens of Gbps at tens of meters [1]. Furthermore, with low latency as well as low energy consumption and highly inherent security, UOWC has been widely appreciated as a more suitable alternative to acoustics in the underwater media. Good amount of work has been published on the model that describes the intensity of laser beam variations to measure statistical properties of so called weak temperature based turbulence's in UOWC channels [24]. UOWC channels with temperature gradients are modeled via generalised Gamma $(\mathrm{gG})$ distribution with an excellent and tight fit to the measured data under all channel conditions as explored in [24]. Decode-and-forward (DF) protocol based on a dual-hop RF-UOWC is analysed in [25]. Findings in the area of co-operative RF-UOWC using hyperbolic-tangent log-normal (HTLN) distribution channel with pointing errors have been presented in [26] that influences the use of a non-commercial radio frequency in underwater technology. Furthermore, to analyse the secrecy performance of a two-hop mixed RF-UOWC system using a DF relay modeled by the $\alpha-\mu$ and exponential-generalised Gamma distribution is evaluated in [27]. More comprehensive work on a unified statistical model for the characterisation of turbulence-induced fading is investigated for UOWC channels based on some experimental work in [10] while it has been extended to a unified framework of a dual-hop UOWC with AF relays in [12]. More recently, a paper on an analysis for improving the performance of a dual-hop RF-UOWC transmission system experiencing a Nakagami- $m$ fading is well studied in [28].

\section{Contributions}

The main contribution of the paper is to thoroughly evaluate the performance of MIMO RF-UOWC E2E system that will play a key role in improving the spectral efficiency of $6^{\text {th }}$ generation (6 G) of cellular networks. Subsequently, the highlights of this work are listed below.

- We assume a system model wherein the transmitter end is equipped with MIMO RF utilising transmit antenna selection (TAS) scheme. This acts as a source $(S)$ communicating with relay $(R)$ via combining the incoming signals utilising maximal ratio combining (MRC) technique at $R$. Subsequently, $R$ communicates with destination $(D)$ via an UOWC link.

- The performance of a mixed dual-hop MIMO RF-UOWC systems is evaluated in-depth wherein the MIMO RF hop is modeled via Nakagami- $m$ fading environment and the UOWC hop is modeled via exponential and generalised Gamma (EGG) distribution, respectively.

- The closed-form expressions for outage probability (OP) and bit error rate (BER) in presence of variable gain relaying (VGR) are derived for different types of detection techniques dependent on $r=1$ (heterodyne) and $r=2$ (intensity modulation / direct detection (IM/DD)).

- Tight asymptotic expressions are derived for OP and average BER applicable in high SNR regimes with negligible computational complexity.

- The accuracy of our derived analytical results are validated via Monte-Carlo simulations. The figures demonstrate results for varying bubbles level and varying temperature gradient. Furthermore, OP and BER results are validated, with respect to both average SNR of MIMO RF hop and electrical SNR of UOWC hop for varying parameters i.e. 


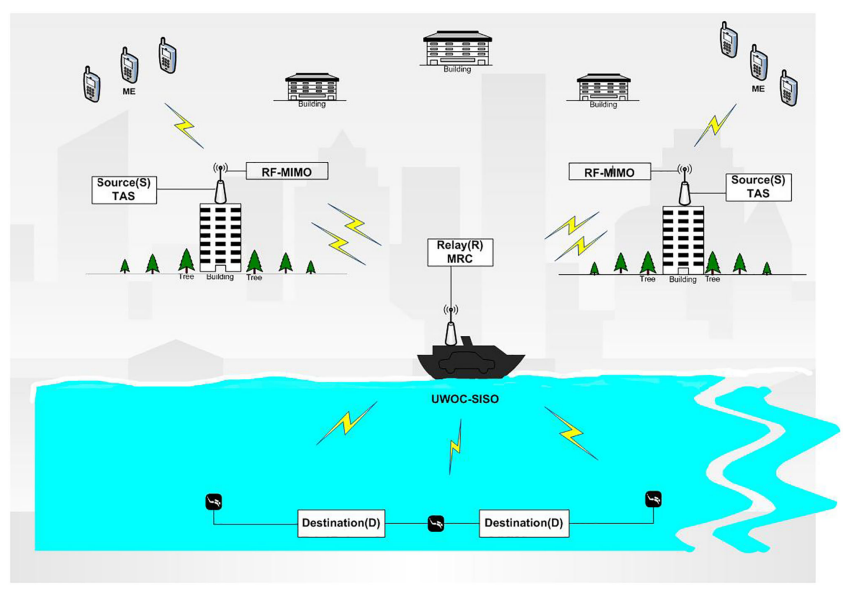

Fig. 1. System model illustrating the MIMO RF and UOWC hops. The source $(S)$ node transmits over MIMO RF channels via $N_{t}$ antennas that is received by $N_{r}$ antennas of the relay $(R)$ node. Subsequently, the $R$ node re-transmits (utilising variable gain relaying technique) that is received by the destination node $(D)$ over an UOWC link.

fading parameters, number of antennas, bubble levels, temperature gradient, and modulation techniques etc.

Overall, to sum up this work, we address the problem of mixed dual-hop MIMO RF-UOWC system via deriving exact and asymptotic closed-form expressions for the OP and BER of various binary modulation schemes. To the best of the authors' knowledge, based on the open literature, such system model is yet an open and unexplored problem.

\section{Organisation}

The remainder of the paper is organised as follows. Section II discusses the system and channel models. Next, Section III presents the statistical characteristics of the E2E system followed by the analytical OP and BER analysis in Section IV. And, finally, Section $\mathrm{V}$ discusses the numerical and simulation results followed by concluding remarks in Section VI.

\section{System Setup AND ChanNel Model}

\section{A. System Setup}

The system model is demonstrated in Fig. 1 with MIMO RF and UOWC hops clearly illustrated in the description. The system under consideration assumes number of antennas for both transmission and reception of signals from $S$ node to $R$ node be denoted by $N_{t}$ and $N_{R}$, respectively. The communication between the $S$ and $R$ nodes takes place over MIMO RF links. The $R$ node assists communication between the $S$ and the $D$ nodes where $R$ node to $D$ node communication is defined via the UOWC channel. The relay operates in full-duplex mode and thus, the time-orthogonal transmission occurs successively.

We denote $S, R$, and $D$ to represent source, relay, and destination nodes, respectively. The channel from $\mathcal{A}$ to $\mathcal{B}$, where $\mathcal{A} \in\{S, R\}, \mathcal{B} \in\{R, D\}$, and $\mathcal{A} \neq \mathcal{B}$, is denoted as $H_{\mathcal{A B}}$. Specifically, $h_{\mathcal{A B}}^{i j}$ is the complex channel from the $i-t h$ transmit antenna at $\mathcal{A}$ to the $j-t h$ receive antenna at $\mathcal{B}$. Prior to data transmission, in each hop, the $S$ node transmits a message to the $R$ node for channel estimation purpose. Subsequently, the $R$ node feeds back the index of the best transmit and receive antenna pairs i.e. the channel that has the maximum absolute magnitude [14]. Utilising this feedback, the transmission and reception of a single pair antenna is randomly selected for the main purpose of data transmission. We assume this feedback is error-free with zero-delay, the complexity of the channel and its absolute magnitude value for the selected transceiver antennas, $\left|h_{\mathcal{A B}}^{\max }\right|$, is defined as

$$
\left|h_{\mathcal{A B}}^{\max }\right|=\max _{i, j}\left\{\left|h_{\mathcal{A B}}^{i, j}\right|\right\} .
$$

We assume $\varepsilon_{S}$ as an average energy available from $S$ node to $R$ node at MIMO RF. Moreover, $\gamma_{S R}=\left|h_{S R}^{\max }\right|^{2} \varepsilon_{S} / N_{o}$ is the instantaneous SNR of the MIMO RF hop, where $N_{o}$ represents the power spectral density of the channel.

In this work, the following two tables from [10] have been utilised that provide an experimental-based understanding on the expectation maximisation (EM) algorithm parameters:

\section{B. Channel Models}

The probability density function (PDF), $\gamma_{S R}$, of MIMO RF hop ( $S$ node to $R$ node) operating under MRC technique is given as [29, eq. (11)]

$$
\begin{aligned}
f_{\gamma_{S R}}(\gamma)= & \frac{N_{t} m^{N_{r} m} \gamma^{N_{r} m-1} e^{\frac{-m \gamma}{\bar{\gamma}} S R}}{\Gamma\left(N_{r} m\right) \bar{\gamma}_{S R} N_{r} m} \\
& \times\left[1-e^{\frac{-m \gamma}{\bar{\gamma}_{S R}}} \sum_{n=0}^{N_{r} m-1} \frac{1}{n !}\left(\frac{m \gamma}{\bar{\gamma}_{S R}}\right)^{n}\right]^{N_{t}-1},
\end{aligned}
$$

where $N_{t}$ is the transmitting antenna, $N_{r}$ is the receiving antenna, $m$ is the Nakagami- $m$ fading channel, $\Gamma($.$) is denoted$ as Gamma function[30, eq. (11.10)], and $\gamma$ is the instantaneous received signal-to-noise ratio (SNR) while $\bar{\gamma}_{S R}$ is the average received SNR at the relay after first hop.

In the first hop, the entries in the channel vector $\mathbf{h}_{S R, i}$ between the $i-t h$ antenna at the $S$ node and $j-t h$ antenna at the $R$ node follow a Nakagami- $m$ distribution with fading parameter $m$. The cumulative distribution function (CDF) of $\gamma_{S R}=\frac{\varepsilon_{S}}{N_{o}}\left\|\mathbf{h}_{S R}\right\|^{2}$ is expressed as [15]

$$
F_{\gamma_{S R}}(\gamma)=1-\frac{\Gamma\left(m N_{r}, \frac{m}{\gamma_{S R}} \gamma\right)}{\Gamma\left(m N_{r}\right)} .
$$

Re-expressing (3), utilising the identity [31, eq. (8.352.2)], for integer values of $m N_{r}$, the CDF of $\gamma_{S R}=\max _{1 \leq i \leq N_{t}}\left\{\gamma_{S R, i}\right\}$ is obtained as

$$
F_{\gamma_{S R}}(\gamma)=\left[1-e^{\frac{-m \gamma}{\bar{\gamma}_{S R}}} \sum_{n=0}^{N_{r} m-1} \frac{1}{n !}\left(\frac{m}{\bar{\gamma}_{S R}} \gamma\right)^{n}\right]^{N_{t}} .
$$

For simplification purpose, the binomial theorem and the power sum expansion, utilising [31, eq. (1.111)] and[32, eq. (9)], is expressed as

$$
\left[\sum_{i=0}^{N_{r} m-1} \frac{1}{i !}\left(\frac{m}{\bar{\gamma}} x\right)^{i}\right]^{n_{0}}=\left\{\sum_{n_{1}=0}^{n_{0}} \sum_{n_{2}=0}^{n_{1}} \ldots \sum_{n_{N_{r} m-1}=0}^{n_{N_{r} m-2}}\right.
$$




$$
\begin{aligned}
& \left.\times \prod_{i-1}^{N_{r} m-1}\left(\begin{array}{c}
n_{i}-1 \\
n_{i}
\end{array}\right)\left(\frac{1}{i !}\right)^{n_{i}-n_{i+1}}\left(\frac{m}{\bar{\gamma}} x\right)^{n_{i}}\right\} \\
& =\prod_{i=1}^{N_{r} m-1}\left[\sum_{n_{i}=0}^{n_{i}-1}\left(\begin{array}{c}
n_{i}-1 \\
n_{i}
\end{array}\right)\left(\frac{1}{i !}\right)^{n_{i}-n_{i+1}}\left(\frac{m}{\bar{\gamma}}\right)^{n_{i}}\right] x^{N},
\end{aligned}
$$

where $N_{r} m>1, n_{N_{r} m}=0$, and $N=n_{1}+n_{2}+n_{3}+\cdots+$ $n_{N_{r} m-1}$. Hence, on applying (6) to (4) while denoting $n_{0}=$ $N_{t}, k=i$, and $x=\gamma$, the simplified CDF for the MIMO RF first hop is obtained as

$$
\begin{aligned}
F_{\gamma_{S R}}(\gamma) & =\sum_{n=0}^{N_{t}}\left(\begin{array}{c}
N_{t} \\
n
\end{array}\right)(-1)^{n} e^{\frac{-n \gamma m}{\bar{\gamma}_{S R}}}\left\{\sum_{n_{1}=0}^{N_{t}} \sum_{n_{2}=0}^{n_{1}} \ldots \sum_{n_{N_{r} m-1}=0}^{n_{N_{r} m-2}}\right. \\
\times & {\left.\left[\prod_{k=1}^{N_{r} m-1}\left(\begin{array}{c}
n_{k-1} \\
n_{k}
\end{array}\right)\left(\frac{1}{k !}\right)^{n_{k}-n_{k+1}}\left(\frac{m}{\bar{\gamma}_{S R}}\right)^{n_{k}} \gamma^{n_{k}}\right]\right\} . }
\end{aligned}
$$

On the other hand, with respect to the second hop, the irradiance fluctuations (IF), $I$, of the received optical wave due to air bubbles and temperature induced fading in both fresh and salty waters can be modeled by the mixture of EGG distribution, which is a weighted sum of the exponential and generalised Gamma distributions that is expressed as [10]

$$
\begin{aligned}
f_{I}(I) & =w f(I, \lambda)+(1-w) g(I ;[a, b, c]), \\
f(I: \lambda) & =\frac{1}{\lambda} e^{-\frac{I}{\lambda}}, \\
g(I ;[a, b, c]) & =c \frac{I^{a c-1}}{b^{a c}} \frac{e^{-\frac{I^{c}}{}}}{\Gamma(a)},
\end{aligned}
$$

where $f($.$) and g($.$) represent the exponential and generalised$ Gamma distributions, respectively. In this work, EM algorithm is utilised to determine the maximum likelihood estimates of the model parameters, i.e. $w, \lambda, a, b$, and $c$, where $w$ is the mixture weight or mixture coefficient of the distributions satisfying $0<w<1, \lambda$ is the parameter associated with the exponential distribution, and $a, b$, and $c$ are parameters of the generalised Gamma distribution. The unified PDF, with respect to two different detection types, of the UOWC links are expressed as [10]

$$
\begin{aligned}
f_{\gamma_{R D}}(\gamma)= & \frac{\omega}{r \gamma} G_{0,1}^{1,0}\left[\frac{1}{\lambda}\left(\frac{\gamma}{\mu_{r}}\right)^{\frac{1}{r}} \mid \begin{array}{c}
- \\
1
\end{array}\right] \\
& +\frac{c(1-\omega)}{r \gamma \Gamma(a)} G_{0,1}^{1,0}\left[\frac{1}{b^{c}}\left(\frac{\gamma}{\mu_{r}}\right)^{\frac{c}{r}} \mid \begin{array}{l}
a \\
a
\end{array} .\right.
\end{aligned}
$$

Subsequently, the CDF, $F_{\gamma}(\gamma)=\int_{0}^{\gamma} f_{\gamma}(\gamma) d \gamma$, is obtained as

$$
\begin{aligned}
F_{\gamma_{R D}}(\gamma)= & \omega G_{1,2}^{1,1}\left[\frac{1}{\lambda}\left(\frac{\gamma}{\mu_{r}}\right)^{\frac{1}{r}} \mid \begin{array}{c}
1 \\
1,0
\end{array}\right] \\
& +\frac{(1-\omega)}{\Gamma(a)} G_{1,2}^{1,1}\left[\frac{1}{b^{c}}\left(\frac{\gamma}{\mu_{r}}\right)^{\frac{c}{r}} \mid \begin{array}{c}
1 \\
a, 0
\end{array}\right],
\end{aligned}
$$

where in the case of heterodyne detection technique, the electrical SNR, $\mu_{1}$, is defined as $\mu_{1}=\bar{\gamma}_{R D}$, while for IM/DD technique, the electrical SNR, $\mu_{2}$, is expressed as [10, eq. (19)]

$$
\mu_{2}=\frac{\bar{\gamma}_{R D}}{2 \omega \lambda^{2}+b^{2}(1-\omega) \Gamma(a+2 / c) / \Gamma(a)},
$$

$\bar{\gamma}_{R D}$ is the average received SNR at the destination after second hop, and $G_{., .}^{.,}\left[{ }_{.,} . . \mid \cdot\right]$ is defined as the Meijer's G function [33, eq. (32)].

\section{END-TO-END STATISTICAL CHARACTERISTIC}

Utilising the formula of VGR, we express the E2E statistical characteristic of our system as [29]

$$
F_{\gamma_{e q}}=\frac{\gamma_{S R} \gamma_{R D}}{\gamma_{S R}+\gamma_{R D}+1}
$$

where $\gamma_{S R}$ and $\gamma_{R D}$ are the instantaneous SNR's for $S$-to- $R$ and $R$-to- $D$ nodes, respectively. Since it is quite complicated, if not impossible, to derive the exact closed-form solution for the CDF of the E2E SNR expressed in (10), we utilise the following tight approximation of the E2E SNR under VGR [1]

$$
F_{\gamma_{e q}} \approx \min \left(\gamma_{S R}, \gamma_{R D}\right)
$$

Given the above approximation, the E2E CDF of the SNR $\gamma_{e q}$ is expressed as

$$
\begin{aligned}
F_{\gamma_{e q}}(\gamma) & \approx \operatorname{Pr}\left[\min \left(\gamma_{S R}, \gamma_{R D}\right)<\gamma\right] \\
& =1-\left(1-F_{\gamma_{S R}}(\gamma)\right)\left(1-F_{\gamma_{R D}}(\gamma)\right) \\
& =F_{\gamma_{S R}}(\gamma)+F_{\gamma_{R D}}(\gamma)-F_{\gamma_{S R}}(\gamma) F_{\gamma_{R D}}(\gamma)
\end{aligned}
$$

Hence, on substituting (7) and (10) into (14), we obtained the E2E CDF of our system as shown in (15), shown at the bottom of the next page.

\section{PERFormance ANALYsis}

\section{A. Outage Probability $(O P)$}

In this Section, we deal with the OP for the defined E2E system. The OP, $P_{\text {out }}$, is defined as the probability that $\gamma_{e q}$ falls below a predefined SNR threshold $\gamma_{t h}$. Therefore, $P_{\text {out }}$ is given as [14, eq. (5)]

$$
P_{\text {out }}=\operatorname{P}_{\mathrm{r}}\left[\gamma_{e q}<\gamma_{t h}\right]=F_{\gamma_{e q}}\left(\gamma_{t h}\right),
$$

Eq. (17), shown at the bottom of the next page, where $F_{\gamma_{e q}}\left(\gamma_{t h}\right)$ refers to the CDF of $\gamma_{e q}$ evaluated at $\gamma=\gamma_{t h}$. Hence, the OP for our system is obtained by (15) with $\gamma=\gamma_{t h}$.

\section{B. Average Bit Error Rate (BER)}

BER is defined as the percentage of bits that have errors relative to the total number of bits received in a transmission. The error rate is expressed as [34, eq. (12)]

$$
P_{e}=\frac{q^{p}}{2 \Gamma(p)} \int_{0}^{\infty} e^{-q \gamma} \gamma^{p-1} F_{\gamma_{e q}}(\gamma) d \gamma
$$

where $p$ and $q$ are the modulation parameters and being considered from the Table III.

Hence, to derive the average BER for our E2E system, we substitute (15) into (18) to obtain

$$
P_{e}=P_{e_{a}}+P_{e_{b}}+P_{e_{c}}+P_{e_{d}}+P_{e_{e}}
$$


TABLE I

PARAMETERS OF THE EGG ON THE ESTIMATED AND MEASURED VALUES FOR THE TEMPERATURE GRADIENT OF AN UOWC SYSTEM

\begin{tabular}{||c|c|c||}
\hline $\begin{array}{c}\text { Bubble Level } \\
\text { BL (L/min) }\end{array}$ & $\begin{array}{c}\text { Temperature Gradient } \\
\left({ }^{\circ} \mathrm{C} \mathrm{cm}^{-} 1\right)\end{array}$ & $\begin{array}{c}\text { Exponential-Generalised Gamma Distribution (EGG) } \\
(w, \lambda, a, b, c)\end{array}$ \\
\hline 2.4 & 0.05 & $(0.2130,0.3291,1.4299,1.1817,17.1984)$ \\
\hline 2.4 & 0.10 & $(0.2108,0.2694,0.6020,1.2795,21.1611)$ \\
\hline 2.4 & 0.15 & $(0.1807,0.1641,0.2334,1.4201,22.5924)$ \\
\hline 2.4 & 0.20 & $(0.1665,0.1207,0.1559,1.5216,22.8754)$ \\
\hline 4.7 & 0.05 & $(0.4589,0.3449,1.0421,1.5768,35.9424)$ \\
\hline 4.7 & 0.10 & $(0.4539,0.2744,0.3008,1.7053,54.1422)$ \\
\hline 16.5 & 0.22 & $(0.6238,0.1094,0.0111,4.4750,105.3550)$ \\
\hline 23.6 & 0.22 & $(0.7210,0.1479,0.0121,7.4189,65.6983)$ \\
\hline
\end{tabular}

where $P_{e_{a}}, P_{e_{b}}, P_{e_{c}}, P_{e_{d}}$, and $P_{e_{e}}$ are derived as follows. Firstly, $P_{e_{a}}$ is extracted as

$$
\begin{aligned}
P_{e_{a}}= & \frac{q^{p}}{2 \Gamma(p)} \int_{0}^{\infty} e^{-q \gamma} \gamma^{p-1} \sum_{n=0}^{N_{t}}\left(\begin{array}{c}
N_{t} \\
n
\end{array}\right)(-1)^{n} e^{\frac{-n \gamma m}{\bar{\gamma}_{S R}}} \\
& \times\left[\sum _ { n _ { 1 } = 0 } ^ { N _ { t } } \sum _ { n _ { 2 } = 0 } ^ { n _ { 1 } } \ldots \sum _ { n _ { N _ { r } m - 1 } = 0 } ^ { n _ { N _ { r } m - 2 } } \left(\prod_{k=1}^{N_{r} m-1}\left(\begin{array}{c}
n_{k-1} \\
n_{k}
\end{array}\right)\right.\right. \\
& \left.\left.\times\left(\frac{1}{k !}\right)^{n_{k}-n_{k+1}}\left(\frac{m}{\bar{\gamma}_{S R}}\right)^{n_{k}} \gamma^{n_{k}}\right)\right] d \gamma .
\end{aligned}
$$

TABLE II

PARAMETERS OF THE EGG ON THE ESTIMATED AND MEASURED VALUES FOR SALTY AND FRESH WATERS (THERMALLY UNIFORM) UOWC SYSTEMS

\begin{tabular}{||c|c||}
\hline $\begin{array}{c}\text { Bubble Level } \\
\text { BL (L/min) }\end{array}$ & $\begin{array}{c}\text { Exponential-Generalised Gamma Distribution (EGG) } \\
(w, \lambda, a, b, c)\end{array}$ \\
\hline \multicolumn{2}{|c|}{ Salty Water } \\
\hline 2.4 & $(0.1770,0.4687,0.7736,1.1372,49.1773)$ \\
\hline 4.7 & $(0.2064,0.3953,0.5307,1.2154,35.7368)$ \\
\hline 7.1 & $(0.4344,0.4747,0.3935,1.4506,77.0245)$ \\
\hline 16.5 & $(0.4951,0.1368,0.0161,3.2033,82.1030)$ \\
\hline \multicolumn{2}{|c|}{ Fresh Water } \\
\hline 2.4 & $(0.1953,0.5273,3.7291,1.0721,30.3214)$ \\
\hline 4.7 & $(0.2109,0.4603,1.2526,1.1501,41.3258)$ \\
\hline 7.1 & $(0.3489,0.4771,0.4319,1.4531,74.3650)$ \\
\hline 16.5 & $(0.5117,0.1602,0.0075,2.9963,216.8356)$ \\
\hline
\end{tabular}

TABLE III

BER PARAMETERS OF BINARY MODULATIONS

\begin{tabular}{||c|c|c|}
\hline BER Modulation Type (bits / sec) & $p$ & $q$ \\
\hline \hline Coherent Binary Frequency Shift Keying (CBFSK) & 0.5 & 0.5 \\
\hline Coherent Binary Phase Shift Keying (CBPSK) & 0.5 & 1 \\
\hline Non-Coherent Binary Frequency Shift Keying (NBFSK) & 1 & 0.5 \\
\hline Differential Binary Phase Shift Keying (DBPSK) & 1 & 1 \\
\hline
\end{tabular}

On applying simple algebraic manipulations, we rearrange the integral as

$$
\begin{aligned}
& F_{\gamma_{e q}}(\gamma)=\sum_{n=0}^{N_{t}}\left(\begin{array}{c}
N_{t} \\
n
\end{array}\right)(-1)^{n} e^{\frac{-n \gamma m}{\bar{\gamma}} S R}\left[\sum_{n_{1}=0}^{N_{t}} \sum_{n_{2}=0}^{n_{1}} \ldots \sum_{n_{N_{r} m-1}=0}^{n_{N_{r} m-2}}\left(\prod_{k=1}^{N_{r} m-1}\left(\begin{array}{c}
n_{k-1} \\
n_{k}
\end{array}\right)\left(\frac{1}{k !}\right)^{n_{k}-n_{k+1}}\left(\frac{m}{\bar{\gamma}_{S R}}\right)^{n_{k}} \gamma^{n_{k}}\right)\right] \\
& +w G_{1,2}^{1,1}\left[\frac{1}{\lambda}\left(\frac{\gamma}{\mu_{r}}\right)^{\frac{1}{r}} \mid \begin{array}{c}
1 \\
1,0
\end{array}\right]+\frac{(1-w)}{\Gamma(a)} G_{1,2}^{1,1}\left[\frac{1}{b^{c}}\left(\frac{\gamma}{\mu_{r}}\right)^{\frac{c}{r}} \mid \begin{array}{c}
1 \\
a, 0
\end{array}\right]-\sum_{n=0}^{N_{t}}\left(\begin{array}{c}
N_{t} \\
n
\end{array}\right)(-1)^{n} e^{\frac{-n \gamma m}{\bar{\gamma}_{S R}}}\left[\sum_{n_{1}=0}^{N_{t}} \sum_{n_{2}=0}^{n_{1}} \ldots \sum_{n_{N_{r} m-1}=0}^{n_{N_{r} m-2}}\right. \\
& \left.\times\left(\prod_{k=1}^{N_{r} m-1}\left(\begin{array}{c}
n_{k-1} \\
n_{k}
\end{array}\right)\left(\frac{1}{k !}\right)^{n_{k}-n_{k+1}}\left(\frac{m}{\bar{\gamma}_{S R}}\right)^{n_{k}} \gamma^{n_{k}}\right)\right] w G_{1,2}^{1,1}\left[\left(\frac{1}{\lambda}\right)\left(\frac{\gamma}{\mu_{r}}\right)^{\frac{1}{r}} \mid \begin{array}{c}
1 \\
1,0
\end{array}\right]-\sum_{n=0}^{N_{t}}\left(\begin{array}{c}
N_{t} \\
n
\end{array}\right)(-1)^{n} e^{\frac{-n \gamma m}{\bar{\gamma}_{S R}}} \\
& \times\left[\sum_{n_{1}=0}^{N_{t}} \sum_{n_{2}=0}^{n_{1}} \ldots \sum_{n_{N_{r} m-1}=0}^{n_{N_{r} m-2}}\left(\prod_{k=1}^{N_{r} m-1}\left(\begin{array}{c}
n_{k-1} \\
n_{k}
\end{array}\right)\left(\frac{1}{k !}\right)^{n_{k}-n_{k+1}}\left(\frac{m}{\bar{\gamma}_{S R}}\right)^{n_{k}} \gamma^{n_{k}}\right)\right] \frac{(1-w)}{\Gamma(a)} G_{1,2}^{1,1}\left[\frac{1}{b^{c}}\left(\frac{\gamma}{\mu_{r}}\right)^{\frac{c}{r}} \mid \begin{array}{c}
1 \\
a, 0
\end{array}\right]
\end{aligned}
$$

$$
\begin{aligned}
& F_{\gamma_{e q}(\gamma)} \cong \sum_{\mu_{r} \gg 1}^{N_{t}}\left(\begin{array}{c}
N_{t} \\
n
\end{array}\right)(-1)^{n} e^{\frac{-n \gamma m}{\bar{\gamma}_{S R}}}\left[\sum_{n_{1}=0}^{N_{t}} \sum_{n_{2}=0}^{n_{1}} \ldots \sum_{n_{N_{r} m-1}=0}^{n_{N_{r} m-2}}\left(\prod_{k=1}^{N_{r} m-1}\left(\begin{array}{c}
n_{k-1} \\
n_{k}
\end{array}\right)\left(\frac{1}{k !}\right)^{n_{k}-n_{k+1}}\left(\frac{m}{\bar{\gamma}_{S R}}\right)^{n_{k}} \gamma^{n_{k}}\right)\right]
\end{aligned}
$$

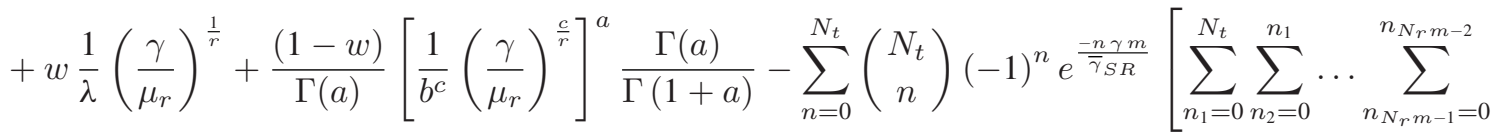

$$
\begin{aligned}
& \left.\times\left(\prod_{k=1}^{N_{r} m-1}\left(\begin{array}{c}
n_{k-1} \\
n_{k}
\end{array}\right)\left(\frac{1}{k !}\right)^{n_{k}-n_{k+1}}\left(\frac{m}{\bar{\gamma}_{S R}}\right)^{n_{k}} \gamma^{n_{k}}\right)\right] w \frac{1}{\lambda}\left(\frac{\gamma}{\mu_{r}}\right)^{\frac{1}{r}}-\sum_{n=0}^{N_{t}}\left(\begin{array}{c}
N_{t} \\
n
\end{array}\right)(-1)^{n} e^{\frac{-n \gamma m}{\bar{\gamma}_{S R}}} \\
& \times\left[\sum_{n_{1}=0}^{N_{t}} \sum_{n_{2}=0}^{n_{1}} \ldots \sum_{n_{N_{r} m-1}=0}^{n_{N_{r} m-2}}\left(\prod_{k=1}^{N_{r} m-1}\left(\begin{array}{c}
n_{k-1} \\
n_{k}
\end{array}\right)\left(\frac{1}{k !}\right)^{n_{k}-n_{k+1}}\left(\frac{m}{\bar{\gamma}_{S R}}\right)^{n_{k}} \gamma^{n_{k}}\right)\right] \frac{(1-w)}{\Gamma(a)}\left[\frac{1}{b^{c}}\left(\frac{\gamma}{\mu_{r}}\right)^{\frac{c}{r}}\right]^{a} \frac{\Gamma(a)}{\Gamma(1+a)}
\end{aligned}
$$




$$
\begin{aligned}
P_{e_{a}}= & \frac{q^{p}}{2 \Gamma(p)} \sum_{n=0}^{N_{t}}\left(\begin{array}{c}
N_{t} \\
n
\end{array}\right)(-1)^{n}\left[\sum_{n_{1}=0}^{N_{t}} \sum_{n_{2}=0}^{n_{1}} \ldots \sum_{n_{N_{r} m-1}=0}^{n_{N_{r} m-2}}\right. \\
& \left.\times\left(\prod_{k=1}^{N_{r} m-1}\left(\begin{array}{c}
n_{k-1} \\
n_{k}
\end{array}\right)\left(\frac{1}{k !}\right)^{n_{k}-n_{k+1}}\left(\frac{m}{\bar{\gamma}_{S R}}\right)^{n_{k}}\right)\right] \\
& \times \int_{0}^{\infty} \gamma^{p-1+\sum_{j=1}^{N_{r} m-1} j} e^{-\left(q+\frac{n m}{\bar{\gamma}_{S R}}\right) \gamma} d \gamma .
\end{aligned}
$$

This integral is solved via utilising[35, eq. (3.35.3)] and we obtained $P_{e_{a}}$ as

$$
\begin{aligned}
P_{e_{a}}= & \frac{q^{p}}{2 \Gamma(p)} \sum_{n=0}^{N_{t}}\left(\begin{array}{c}
N_{t} \\
n
\end{array}\right)(-1)^{n}\left[\sum_{n_{1}=0}^{N_{t}} \sum_{n_{2}=0}^{n_{1}} \ldots \sum_{n_{N_{r} m-1}=0}^{n_{N_{r} m-2}}\right. \\
& \left.\times\left(\prod_{k=1}^{N_{r} m-1}\left(\begin{array}{c}
n_{k-1} \\
n_{k}
\end{array}\right)\left(\frac{1}{k !}\right)^{n_{k}-n_{k+1}}\left(\frac{m}{\bar{\gamma}_{S R}}\right)^{n_{k}}\right)\right] \\
& \times\left(q+\frac{n m}{\bar{\gamma}_{S R}}\right)^{p-2-\sum_{j=1}^{N_{r} m-1} j} \Gamma\left(p+\sum_{j=1}^{N_{r} m-1} j\right) .
\end{aligned}
$$

Secondly, $P_{e_{b}}$ is extracted as

$$
P_{e_{b}}=\frac{q^{p}}{2 \Gamma(p)} w \int_{0}^{\infty} \gamma^{p-1} e^{-q \gamma} G_{1,2}^{1,1}\left[\frac{1}{\lambda}\left(\frac{\gamma}{\mu_{r}}\right)^{\frac{1}{r}} \mid \begin{array}{c}
1 \\
1,0
\end{array}\right] d \gamma
$$

To solve this integral in (23), we convert the exponential function into Meijer's G function via utilising [36, eq. (11)] as $e^{-q \gamma}=$ $G_{0,1}^{1,0}\left[\left.q \gamma\right|_{0}\right]$ and applying [36, eq. (21)], we obtain

$$
P_{e_{b}}=\frac{w}{\Gamma(p)} \sqrt{\frac{\pi^{1-r} r}{2^{r+1}}} G_{r+1,2 r}^{r, r+1}\left[\frac{(\lambda r)^{-r}}{q \mu_{r}} \mid \begin{array}{c}
\Delta(r, 1), 1-p \\
\Delta(r, 1), \Delta(r, 0)
\end{array}\right]
$$

where $\Delta(k, a)=\frac{a}{k}, \frac{a+1}{k}, \ldots, \frac{a+k-1}{k}$. Thirdly, $P_{e_{c}}$ is extracted as

$$
P_{e_{c}}=\frac{q^{p}(1-w)}{2 \Gamma(p) \Gamma(a)} \int_{0}^{\infty} \gamma^{p-1} e^{-q \gamma} G_{1,2}^{1,1}\left[\left.\frac{1}{b^{c}}\left(\frac{\gamma}{\mu_{r}}\right)^{\frac{c}{r}}\right|_{a, 0} ^{1}\right] d \gamma
$$

On utilising similar methodology as applied in obtaining $P_{e_{b}}$, $P_{e_{c}}$ is obtained as

$$
\begin{aligned}
P_{e_{c}}= & \frac{(1-w)}{2 \Gamma(p) \Gamma(a)} \sqrt{\frac{r^{2 a-1} c^{2 p-1}}{(2 \pi)^{c+r-2}}} \\
& \times G_{r+c, 2 r}^{r, r+c}\left[\frac{\left(q^{-1} c\right)^{c}}{\left(b^{c} r\right)^{r} \mu_{r}^{c}} \mid \begin{array}{c}
\Delta(r, 1), \Delta(c, 1-p) \\
\Delta(r, a), \Delta(r, 0)
\end{array}\right] .
\end{aligned}
$$

Fourthly, $P_{e_{d}}$ is extracted as

$$
\begin{aligned}
& P_{e_{d}}=-\frac{q^{p} w}{2 \Gamma(p)} \sum_{n=0}^{N_{t}}\left(\begin{array}{c}
N_{t} \\
n
\end{array}\right)(-1)^{n} \int_{0}^{\infty} \gamma^{p-1} e^{-\left(q+\frac{n m}{\gamma_{S R}}\right) \gamma} \\
& \times\left[\sum _ { n _ { 1 } = 0 } ^ { N _ { t } } \sum _ { n _ { 2 } = 0 } ^ { n _ { 1 } } \ldots \sum _ { n _ { N _ { r } m - 1 } = 0 } ^ { n _ { N _ { r } m - 2 } } \left(\prod_{k=1}^{N_{r} m-1}\left(\begin{array}{c}
n_{k-1} \\
n_{k}
\end{array}\right)\left(\frac{1}{k !}\right)^{n_{k}-n_{k+1}}\right.\right. \\
& \left.\left.\times\left(\frac{m}{\bar{\gamma}_{S R}}\right)^{n_{k}} \gamma^{n_{k}}\right)\right] G_{1,2}^{1,1}\left[\frac{1}{\lambda}\left(\frac{\gamma}{\mu_{r}}\right)^{\frac{1}{r}} \mid \begin{array}{c}
1 \\
1,0
\end{array}\right] d \gamma .
\end{aligned}
$$

Utilising similar algebraic manipulation as applied in (20) to obtain (21), (27) is rewritten as

$$
\begin{aligned}
& P_{e_{d}}=-\frac{q^{p} w}{2 \Gamma(p)} \sum_{n=0}^{N_{t}}\left(\begin{array}{c}
N_{t} \\
n
\end{array}\right)(-1)^{n}\left[\sum_{n_{1}=0}^{N_{t}} \sum_{n_{2}=0}^{n_{1}} \ldots \sum_{n_{N_{r} m-1}=0}^{n_{N_{r} m-2}}\right. \\
& \left.\times\left(\prod_{k=1}^{N_{r} m-1}\left(\begin{array}{c}
n_{k-1} \\
n_{k}
\end{array}\right)\left(\frac{1}{k !}\right)^{n_{k}-n_{k+1}}\left(\frac{m}{\bar{\gamma}_{S R}}\right)^{n_{k}}\right)\right] \\
& \times \int_{0}^{\infty} \gamma^{p-1+\sum_{j=1}^{N_{r} m-1} j} e^{-\left(q+\frac{n m}{\bar{\gamma}_{S R}}\right) \gamma} G_{1,2}^{1,1}\left[\frac{1}{\lambda}\left(\frac{\gamma}{\mu_{r}}\right)^{\frac{1}{r}} \mid \begin{array}{c}
1 \\
1,0
\end{array}\right] d \gamma .
\end{aligned}
$$

Now, on utilising similar methodology as applied to obtain $P_{e_{b}}$, $P_{e_{d}}$ is obtained as presented in (29), shown at the bottom of the next page. Finally, $P_{e_{e}}$ is extracted as

$$
\begin{aligned}
& P_{e_{e}}=-\frac{q^{p}(1-w)}{2 \Gamma(p) \Gamma(a)} \sum_{n=0}^{N_{t}}\left(\begin{array}{c}
N_{t} \\
n
\end{array}\right)(-1)^{n} \int_{0}^{\infty} \gamma^{p-1} e^{-\left(q+\frac{n m}{\bar{\gamma}_{S R}}\right) \gamma} \\
& \times\left[\sum _ { n _ { 1 } = 0 } ^ { N _ { t } } \sum _ { n _ { 2 } = 0 } ^ { n _ { 1 } } \ldots \sum _ { n _ { N _ { r } m - 1 } = 0 } ^ { n _ { N _ { r } m - 2 } } \left(\prod_{k=1}^{N_{r} m-1}\left(\begin{array}{c}
n_{k-1} \\
n_{k}
\end{array}\right)\left(\frac{1}{k !}\right)^{n_{k}-n_{k+1}}\right.\right. \\
& \left.\left.\times\left(\frac{m}{\bar{\gamma}_{S R}}\right)^{n_{k}} \gamma^{n_{k}}\right)\right] G_{1,2}^{1,1}\left[\frac{1}{b^{c}}\left(\frac{\gamma}{\mu_{r}}\right)^{\frac{c}{r}} \mid \begin{array}{c}
1 \\
a, 0
\end{array}\right] d \gamma .
\end{aligned}
$$

Subsequently, employing and implementing similar methodology as was applied to obtain $P_{e_{d}}, P_{e_{e}}$ is obtained as presented in (31), shown at the bottom of next page. Therefore, on combining (22), (24), (26), (29), and (31), we obtain the average BER. See Eq. (32), shown at the bottom of the next page.

\section{NumericAl AND SimUlation RESUltS}

This section reflects on the results derived in this work via Monte-Carlo simulations classifying the importance of OP and BER for a dual-hop E2E system. The results generated for OP and BER with respect to average and/or electrical SNR validating outcomes while exploring the important effects of varying bubble levels $(\mathrm{BL})(\mathrm{L} / \mathrm{min})$, temperature gradients (TG) $\left({ }^{\circ} \mathrm{C} \mathrm{cm}^{-1}\right)$, fresh and saline waters, and binary modulation techniques for the considered E2E system. Moreover, $\mathrm{OP}$ and BER for the considered system have been validated under $m=2$ condition with varying number of antennas at transmitter and receiver as $N_{t}=N_{r}=2,3,4$ or more while altering the parameter $r$ representing both types of detection techniques. 
In Figs. 2 and 3, varying BLs validate the behaviour of the E2E statistics of our proposed system.

The number of antennas are fixed in first hop i.e. $N_{t}=N_{r}=$ 4. It is clearly noticed that as the SNR increases, the behaviour of signals in UOWC changes with an increase in the BL, as discussed in [10] and [12]. One of the primary causes is variations in the refractive index along the path of the optical field traversing the water medium, due to change in air bubbles, temperature, and salinity, resulting in signal fading that impairs the UOWC system performance. Herein, increasing the $\mathrm{BL}$ from $16.5(\mathrm{~L} / \mathrm{min})$ to $23.6(\mathrm{~L} / \mathrm{min})$ experiences a greater $\mathrm{OP}$ at a desired average SNR. Specifically, OP with $r=1$ outperforms that with $r=2$, which means heterodyne technique obtains better performance compared with IM/DD technology. Additionally, it is noticed

$$
\begin{aligned}
P_{e_{d}}= & -\frac{q^{p} w}{2 \Gamma(p)} \sum_{n=0}^{N_{t}}\left(\begin{array}{c}
N_{t} \\
n
\end{array}\right)(-1)^{n}\left[\sum_{n_{1}=0}^{N_{t}} \sum_{n_{2}=0}^{n_{1}} \ldots \sum_{n_{N_{r}} m-1}^{n_{N_{r} m-2}}\left(\prod_{k=1}^{N_{r} m-1}\left(\begin{array}{c}
n_{k-1} \\
n_{k}
\end{array}\right)\left(\frac{1}{k !}\right)^{n_{k}-n_{k+1}}\left(\frac{m}{\bar{\gamma}_{S R}}\right)^{n_{k}}\right)\right] \\
& \times \sqrt{\frac{r}{(2 \pi)^{r-1}}}\left(q+\frac{n m}{\bar{\gamma}_{S R}}\right)^{-\left(p+\sum_{j=1}^{N_{r} m-1} j\right)} G_{r+1,2 r}^{r, r+1}\left[\frac{(\lambda r)^{-r}}{\left(q+\frac{n m}{\bar{\gamma}_{S R}}\right) \mu_{r}} \mid \begin{array}{c}
\Delta(r, 1), 1-p-\sum_{j=1}^{N_{r} m-1} j \\
\Delta(r, 1), \Delta(r, 0)
\end{array}\right]
\end{aligned}
$$

$$
\begin{aligned}
& P_{e_{e}}=-\frac{q^{p}(1-w)}{2 \Gamma(p) \Gamma(a)} \sum_{n=0}^{N_{t}}\left(\begin{array}{c}
N_{t} \\
n
\end{array}\right)(-1)^{n}\left[\sum_{n_{1}=0}^{N_{t}} \sum_{n_{2}=0}^{n_{1}} \ldots \sum_{n_{N_{r} m-1}=0}^{n_{N_{r} m-2}}\left(\prod_{k=1}^{N_{r} m-1}\left(\begin{array}{c}
n_{k-1} \\
n_{k}
\end{array}\right)\left(\frac{1}{k !}\right)^{n_{k}-n_{k+1}}\left(\frac{m}{\bar{\gamma}_{S R}}\right)^{n_{k}}\right)\right]
\end{aligned}
$$

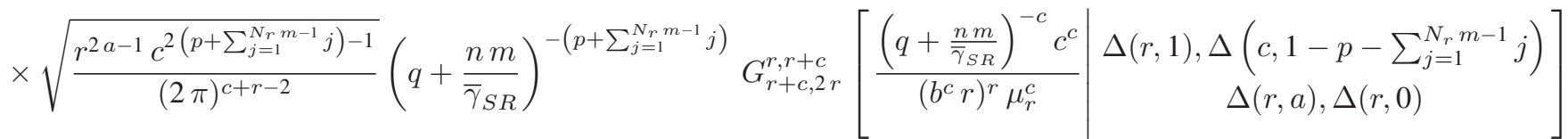

$$
\begin{aligned}
& \left.P_{e} \cong \frac{q^{p}}{\mu_{r} \gg 1} \sum_{n=0}^{N_{t}}\left(\begin{array}{c}
N_{t} \\
n
\end{array}\right)(-1)^{n}\left[\sum_{n_{1}=0}^{N_{t}} \sum_{n_{2}=0}^{n_{1}} \ldots \sum_{n_{N_{r} m-1}=0}^{n_{N_{r} m-2}}\left(\prod_{k=1}^{N_{r} m-1}\left(\begin{array}{c}
n_{k-1} \\
n_{k}
\end{array}\right)\left(\frac{1}{k !}\right)^{n_{k}-n_{k+1}}\left(\frac{m}{\bar{\gamma}_{S R}}\right)^{n_{k}}\right)\right] \Gamma\left(p+\sum_{j=1}^{N^{N_{r}}}\right)^{m-1}\right)^{m} \\
& \times\left(q+\frac{n m}{\bar{\gamma}_{S R}}\right)^{p-2-\sum_{j=1}^{N_{r} m-1} j}+\frac{w}{\Gamma(p)} \sqrt{\frac{\pi^{1-r} r}{2^{r+1}}} \sum_{k=1}^{r}\left[\frac{\left(q \mu_{r}\right)^{-\frac{1}{r}}}{\lambda r}\right]^{k} \frac{\Gamma\left(p+\frac{k}{r}\right) \prod_{l=1 ; l \neq k}^{r} \Gamma\left(\frac{l-k}{r}\right) \prod_{l=1}^{r} \Gamma\left(1+\frac{k-l}{r}\right)}{\prod_{l=r+1}^{2 r} \Gamma\left(2+\frac{1-l+k}{r}\right)} \\
& +\frac{(1-w)}{2 \Gamma(p) \Gamma(a)} \sqrt{\frac{r^{2 a-1} c^{2 p-1}}{(2 \pi)^{c+r-2}}} \sum_{k=1}^{r}\left[\frac{\mu_{r}^{c}\left(b^{c} r\right)^{r}}{\left(q^{-1} c\right)^{c}}\right]^{\frac{1-a-k}{r}} \frac{\prod_{l=1 ; l \neq k}^{r} \Gamma\left(\frac{l-k}{r}\right) \prod_{l=1}^{r} \Gamma\left(1+\frac{a+k-1-l}{r}\right) \prod_{l=r+1}^{r+c} \Gamma\left(1+\frac{p+r-l}{c}+\frac{a+k-1}{r}\right)}{\prod_{l=r+1}^{2 r} \Gamma\left(2+\frac{k+a-l}{r}\right)} \\
& -\frac{q^{p} w}{2 \Gamma(p)} \sum_{n=0}^{N_{t}}\left(\begin{array}{c}
N_{t} \\
n
\end{array}\right)(-1)^{n}\left[\sum_{n_{1}=0}^{N_{t}} \sum_{n_{2}=0}^{n_{1}} \ldots \sum_{n_{N_{r} m-1}=0}^{n_{N_{r} m-2}}\left(\prod_{k=1}^{N_{r} m-1}\left(\begin{array}{c}
n_{k-1} \\
n_{k}
\end{array}\right)\left(\frac{1}{k !}\right)^{n_{k}-n_{k+1}}\left(\frac{m}{\bar{\gamma}_{S R}}\right)^{n_{k}}\right)\right] \sqrt{\frac{r}{(2 \pi)^{r-1}}}
\end{aligned}
$$

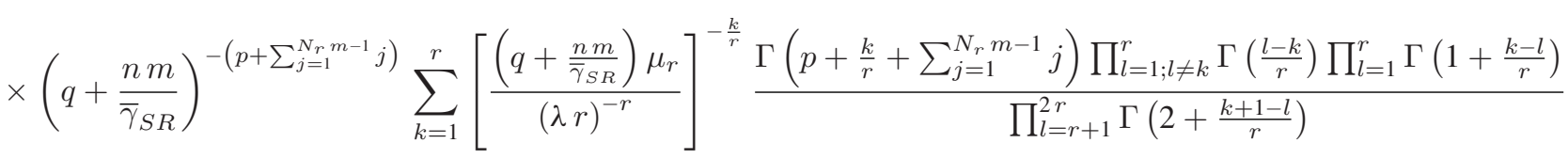

$$
\begin{aligned}
& -\frac{q^{p}(1-w)}{2 \Gamma(p) \Gamma(a)} \sum_{n=0}^{N_{t}}\left(\begin{array}{c}
N_{t} \\
n
\end{array}\right)(-1)^{n}\left[\sum_{n_{1}=0}^{N_{t}} \sum_{n_{2}=0}^{n_{1}} \ldots \sum_{n_{N_{r} m-1}=0}^{n_{N_{r} m-2}}\left(\prod_{k=1}^{N_{r} m-1}\left(\begin{array}{c}
n_{k-1} \\
n_{k}
\end{array}\right)\left(\frac{1}{k !}\right)^{n_{k}-n_{k+1}}\left(\frac{m}{\bar{\gamma}_{S R}}\right)^{n_{k}}\right)\right] \\
& \times \sqrt{\frac{r^{2 a-1} c^{2\left(p+\sum_{j=1}^{N_{r} m-1} j\right)-1}}{(2 \pi)^{c+r-2}}}\left(q+\frac{n m}{\bar{\gamma}_{S R}}\right)^{-\left(p+\sum_{j=1}^{N_{r} m-1} j\right)} \sum_{k=1}^{r}\left[\frac{\left(b^{c} r\right)^{r} \mu_{r}^{c}}{\left(q+\frac{n m}{\bar{\gamma}_{S R}}\right)^{-c} c^{c}}\right]^{\frac{1-a-k}{r}} \\
& \times \frac{\prod_{l=1 ; l \neq k}^{r} \Gamma\left(\frac{l-k}{r}\right) \prod_{l=1}^{r} \Gamma\left(1+\frac{a+k-1-l}{r}\right) \prod_{l=r+1}^{r+c} \Gamma\left(1+\frac{p+r-l+\sum_{j=1}^{N r m-1} j}{c}+\frac{a+k-1}{r}\right)}{(-2)} \\
& \prod_{l=r+1}^{2 r} \Gamma\left(2+\frac{k+a-l}{r}\right)
\end{aligned}
$$




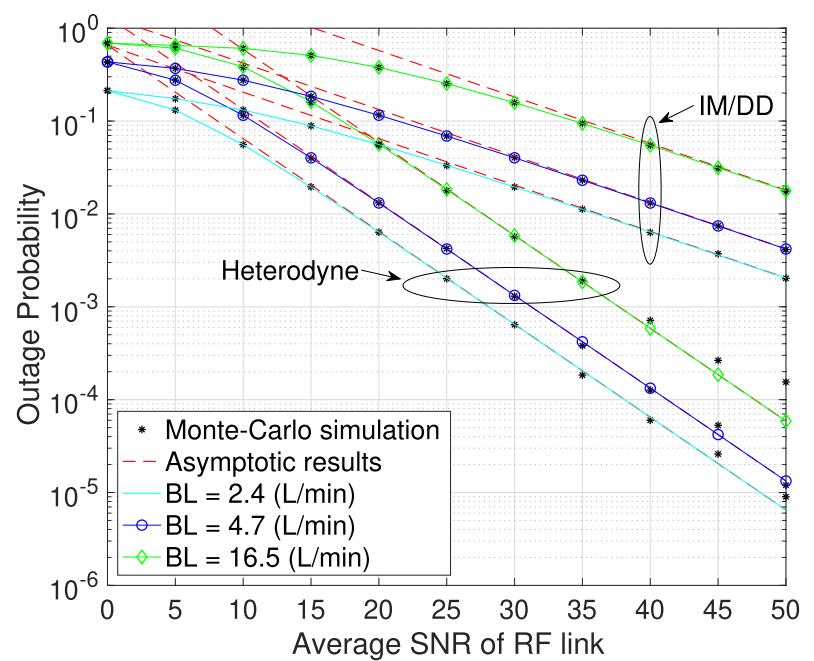

Fig. 2. Outage probability with respect to average SNR of S-R RF link for varying bubble levels under both detection types (i.e. varying $r$ ) of the R-D UOWC link along with asymptotes.

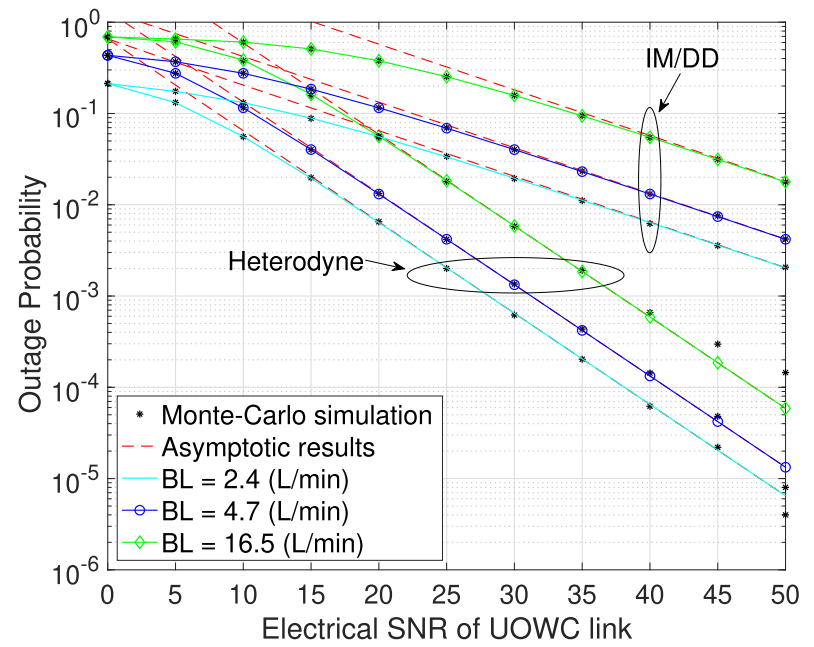

Fig. 3. Outage Probability with respect to electrical SNR of UOWC of S-R RF link for varying Bubble Level under both detection types (i.e. varying $r$ ) of the R-D UOWC link along with asymptotes.

from Fig. 2 that the Monte Carlo simulations perfectly match with the analytical results confirming the accuracy of our derived analysis.

Figs. 4 and 5 demonstrate the altering behaviour of the BLs for both fresh and salty waters while keeping other parameters fixed as provided in Table II.

The change in OP with respect to average SNR of RF hop for varying BLs in fresh and salty waters provides an invaluable understanding on how the behaviour of salinity effects the signal levels in underwater. Again this accurate the tightness of the derived asymptotic expression.

In Figs. 6 and 7, we observe the varying characteristics of the OP for average SNR of RF hop and for electrical SNR of UOWC hop, respectively, with varying TGs in presence of both types of detection techniques in the UOWC channel.

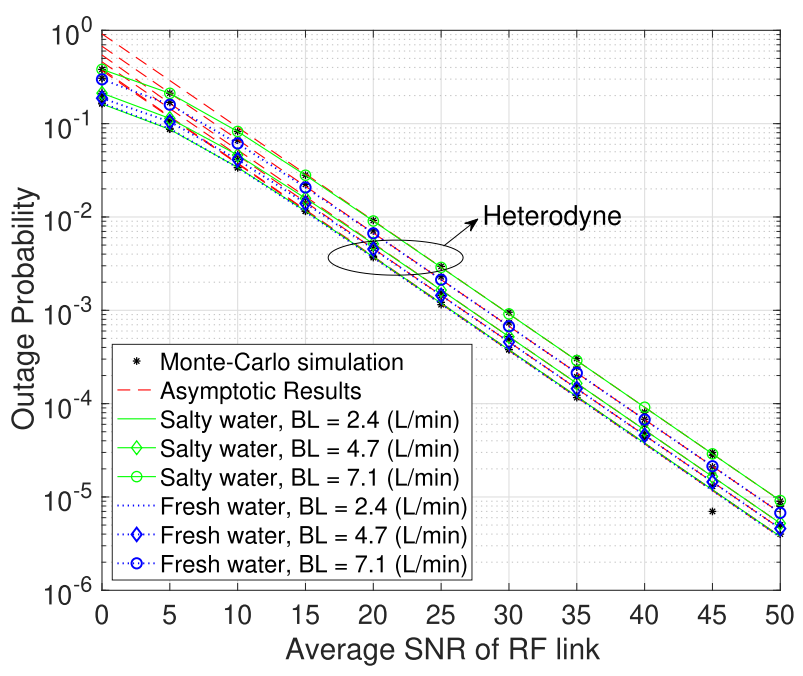

Fig. 4. Outage Probability with respect to average SNR of S-R RF link for varying bubble levels under salty and fresh waters employing the R-D UOWC link along with asymptotes.

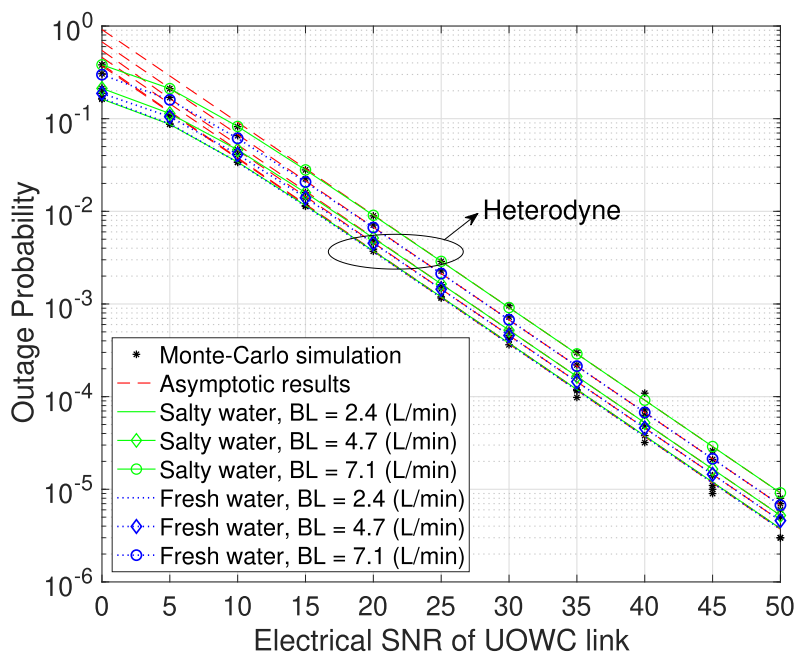

Fig. 5. Outage probability with respect to electrical SNR of UOWC of R-D link for varying bubble levels in salty and fresh waters along with asymptotes.

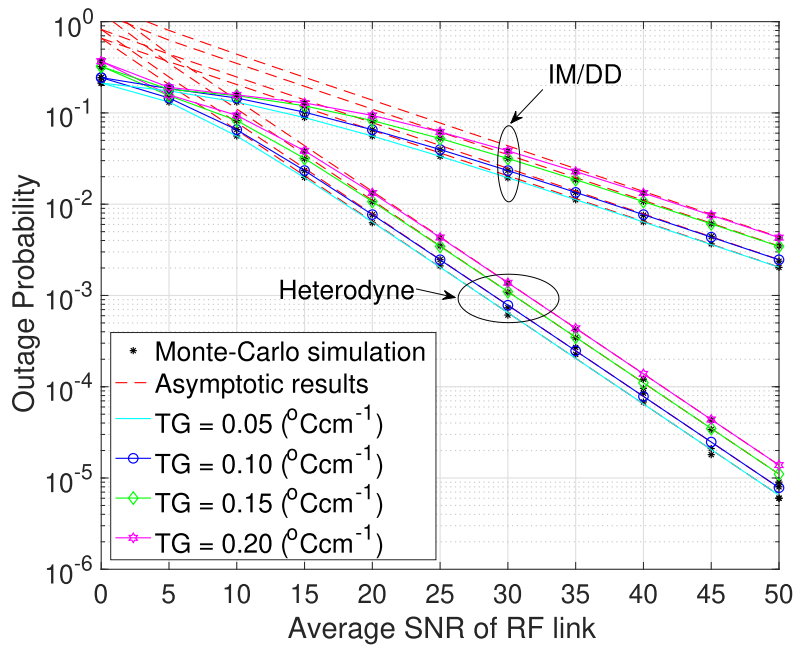

Fig. 6. Outage probability with respect to average SNR of S-R RF link for varying temperature gradients under both detection types (i.e. varying $r$ ) of the R-D UOWC link along with asymptotes. 


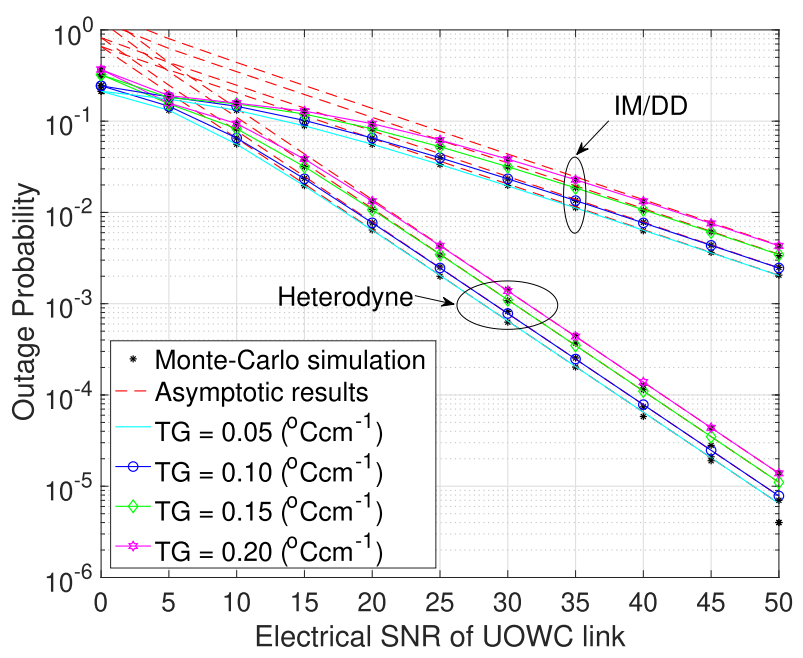

Fig. 7. Outage probability with respect to electrical SNR of UOWC of R-D link for varying temperature gradients along with asymptotes.

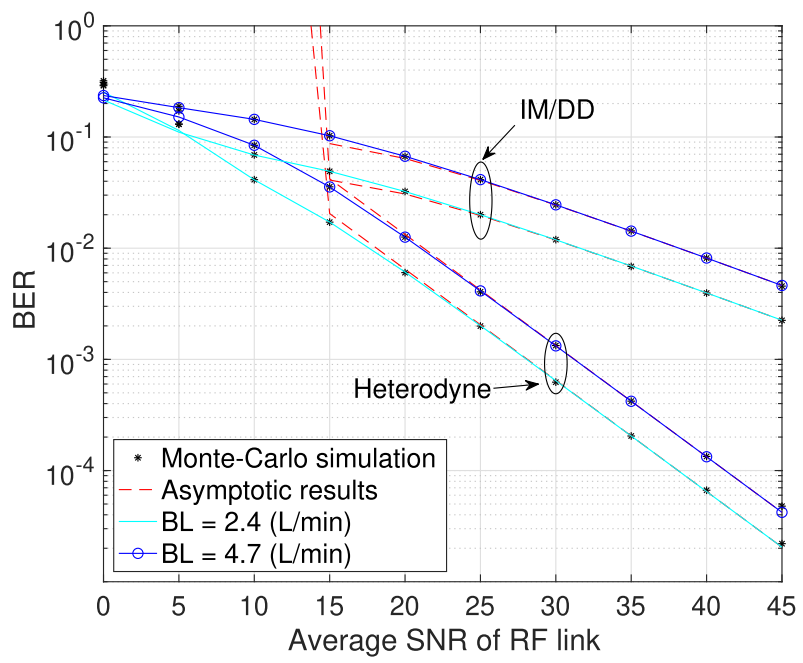

Fig. 8. Bit error rate with respect to average SNR of S-R RF links for varying bubble levels under both detection types (i.e. varying $r$ ) of the R-D UOWC link along with asymptotes.

Temperatures at $0.05\left({ }^{\circ} \mathrm{C} \mathrm{cm}^{-1}\right)$ for the heterodyne detection technique perform better with respect to increasing temperature, i.e. $0.10\left({ }^{\circ} \mathrm{C} \mathrm{cm}^{-1}\right), 0.15\left({ }^{\circ} \mathrm{C} \mathrm{cm}^{-1}\right)$, and $0.20\left({ }^{\circ} \mathrm{C} \mathrm{cm}^{-1}\right)$. It has been noticed that there is a slight variation in the temperature curves for increasing level of temperature. And, temperature in the UOWC effects the turbulence's, which results in the variation of its OP.

Furthermore, the asymptotic results of outage probability at high SNR regime obtained in (17) is also demonstrated in Figs. 2 through 7. As can be clearly observed from these figures, the asymptotic results of the outage probability is in close tightness with analytical results in high SNR regime. This justifies the accuracy and tightness of this derived asymptotic expression.

In Fig. 8, BER results are plotted against average SNR of RF hop for varying BLs applicable to the considered E2E system. We have noticed a promising response for varying BLs from $2.4(\mathrm{~L} / \mathrm{min})$ to $4.7(\mathrm{~L} / \mathrm{min})$.

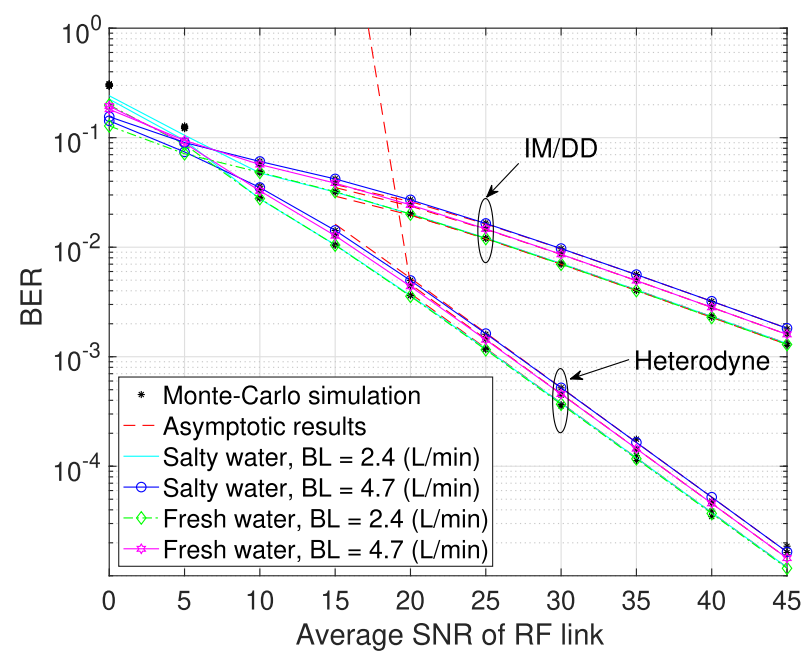

Fig. 9. Bit error rate with respect to average SNR of S-R RF links for varying bubble levels of salty and fresh water under both detection types (i.e. varying $r$ ) of the R-D UOWC link along with asymptotes.

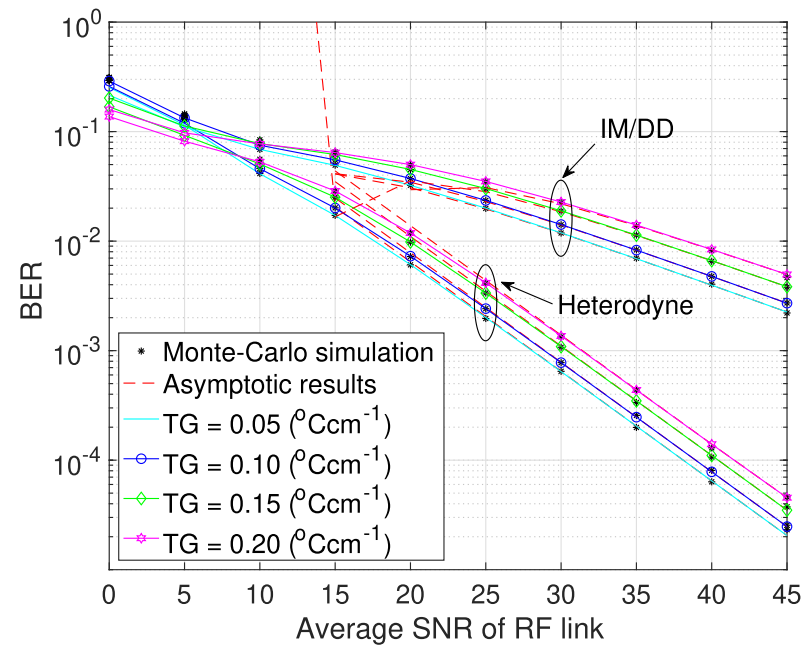

Fig. 10. Bit error rate with respect to average SNR of S-R RF links for varying temperature gradients under both detection types (i.e. varying $r$ ) of the R-D UOWC link along with asymptotes.

In Fig. 9, the plots demonstrate the changing behaviour of varying BLs for fresh and salty water while keeping all the other parameters constant i.e. $N_{t}=N_{r}=4$ and $m=2$ etc. The change in BER vs average SNR of RF hop for varying BLs from fresh to salty waters gives us an understanding on the fact that behaviour of salinity effects the signal levels. The BER vs average SNR of RF hop under the fresh and salty waters employing both types of detection techniques of UOWC hop for the E2E system are plotted. Clearly for fresh and salty waters, fresh water with $B L=2.4(\mathrm{~L} / \mathrm{min})$ behaves better than salty water scenario for the same $B L=2.4(\mathrm{~L} / \mathrm{min})$. It demonstrates slight variations in the behaviour of BER under $B L=4.7(\mathrm{~L} / \mathrm{min})$ for fresh and salty waters.

In Fig. 10, plots are based on the BER versus average SNR of RF hop for varying TGs while keeping $B L=2.4(\mathrm{~L} / \mathrm{min})$ constant. We have noticed a promising response in varying TGs 


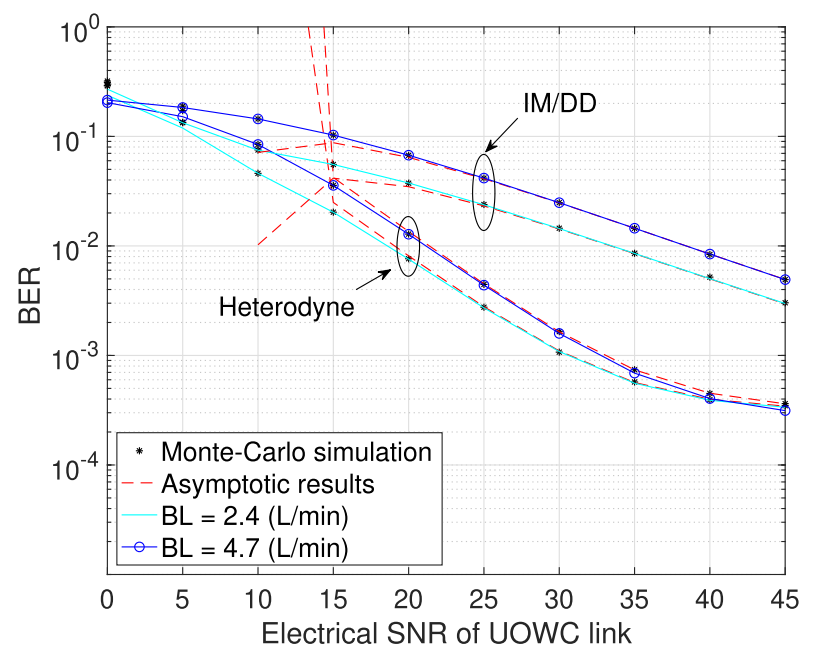

Fig. 11. Bit error rate with respect to electrical SNR of UOWC of R-D link for varying bubble levels under both detection types (i.e. varying $r$ ) of the R-D UOWC link along with asymptotes.

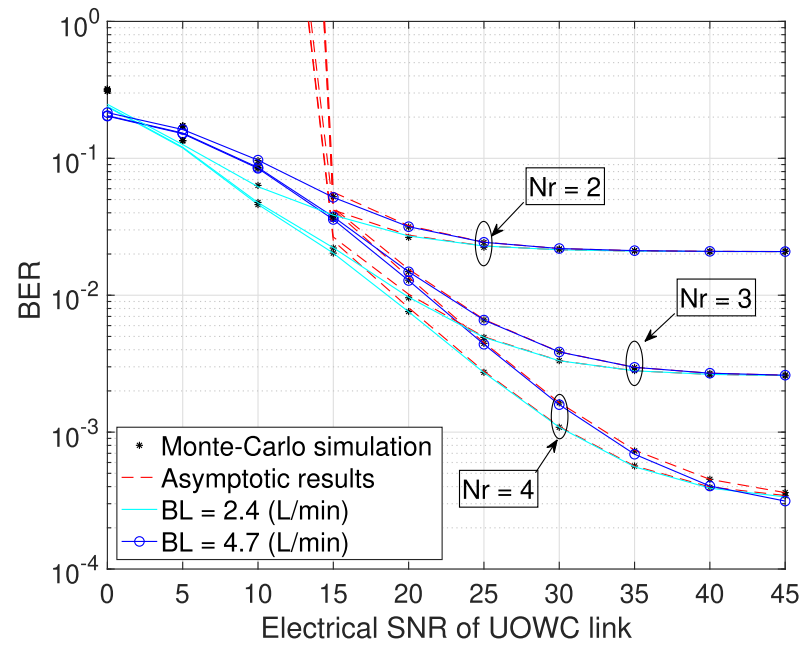

Fig. 12. Bit error rate with respect to electrical SNR of UOWC link with varying $N_{r}$ of S-R RF links and varying bubble levels of the R-D UOWC link along with asymptotes.

from $0.05\left({ }^{\circ} \mathrm{C} \mathrm{cm}^{-1}\right)$ to $0.20\left({ }^{\circ} \mathrm{C} \mathrm{cm}^{-1}\right)$. The behaviour of BER plays an important role in providing valuable insights based on how well we take into account the BL ranges for various scenarios.

In Fig. 11, plots are based on the BER versus electrical SNR of UOWC link for varying $B L=2.4(\mathrm{~L} / \mathrm{min})$ and $4.7(\mathrm{~L} / \mathrm{min})$ applicable to both types of detection techniques based on $r$. It has been noticed that an increase in electrical SNR does not improves performance since the average SNR of RF hop acts as a bottleneck thereby leading to a floor in the curves for the heterodyne technique.

In Figs. 12 and 13, the results are based on the BER versus electrical SNR for UOWC hop with varying $B L=2.4(\mathrm{~L} / \mathrm{min})$ and $4.7(\mathrm{~L} / \mathrm{min})$ and varying $T G=0.05\left({ }^{\circ} \mathrm{C} \mathrm{cm}^{-1}\right)$ to $T G=$ $0.15\left({ }^{\circ} \mathrm{C} \mathrm{cm}^{-1}\right)$, respectively, besides varying numbers of antennas for the receiver $N_{r}$ at first hop $(S-R)$.

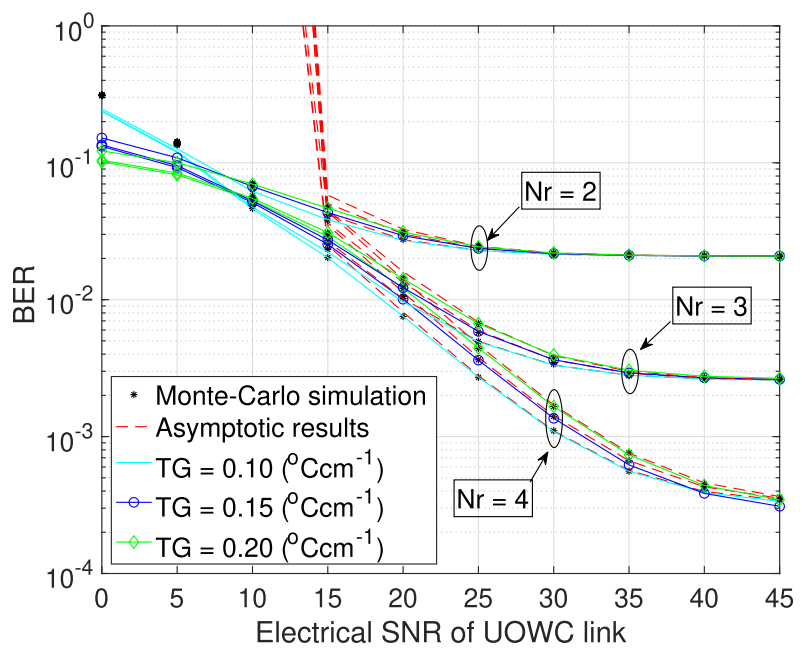

Fig. 13. Bit error rate with respect to electrical SNR of UOWC link with varying $N_{r}$ (S-R) RF links for varying temperature gradients along with asymptotes.

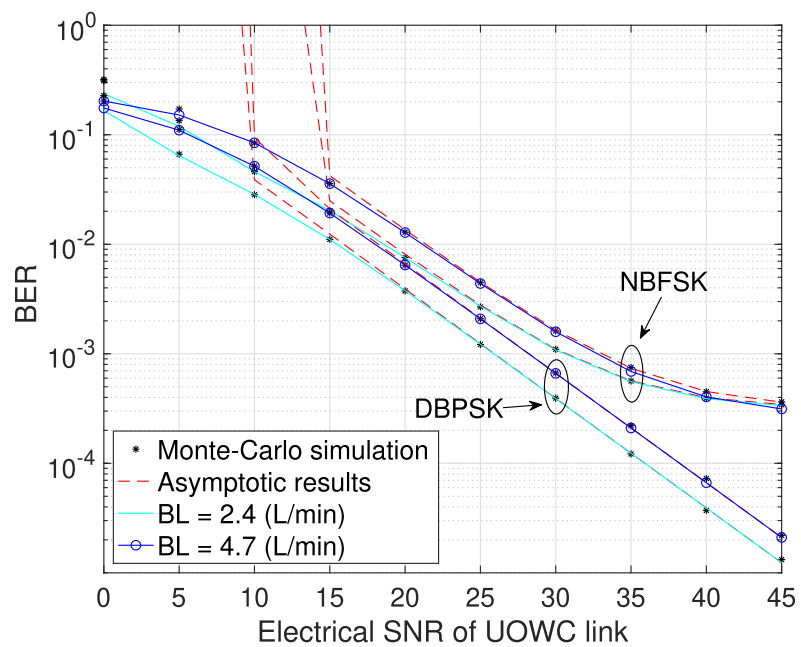

Fig. 14. Bit error rate with respect to electrical SNR of UOWC link for different modulation schemes based on varying $q$ besides varying bubble levels of the R-D UOWC link along with asymptotes.

Herein, increase in the number of antennas at the S-R hop outperforms the lesser number of antennas as can be observed in both the figures with respect to BLs and TGs thereby demonstrating a promising response for an increase in the number of antennas that can be ultimately applied to the real time scenarios. It has been noticed for the number of antennas that an increase in electrical SNR does not improve the performance since the average SNR of RF hop acts as a bottleneck thereby leading to a floor in the curves for $N_{r}=2,3,4$ at the RF hop.

Lastly, in Figs. 14 and 15, our results are based on the BER versus electrical SNR of UOWC link with varying $B L=2.4(\mathrm{~L} / \mathrm{min})$ and $B L=4.7(\mathrm{~L} / \mathrm{min})$ and varying $T G=$ $0.05\left({ }^{\circ} \mathrm{C} \mathrm{cm}^{-1}\right)$ to $T G=0.20\left({ }^{\circ} \mathrm{C} \mathrm{cm}^{-1}\right)$, respectively, involving different binary modulation schemes.

DBPSK modulation technique scenarios perform better relative to NBFSK counterparts. This demonstrates a promising 


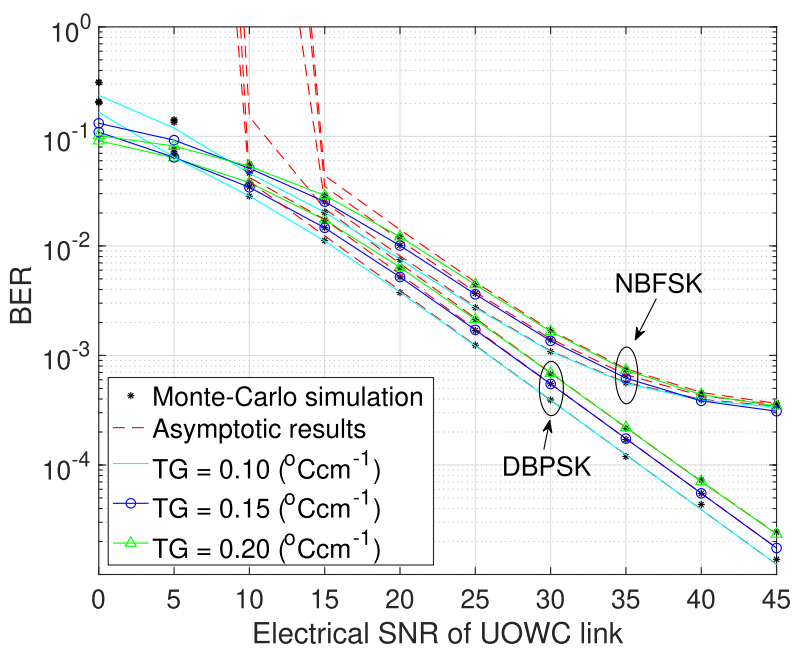

Fig. 15. Bit error rate with respect to electrical SNR of UOWC link with different modulation schemes based on varying $q$ besides varying temperature gradients of the R-D UOWC link along with asymptotes.

behaviour for the binary modulation schemes involved in dualhop systems as employed in our proposed system. Once again, it has been noticed that an increase in electrical SNR does not improve the performance as the average SNR of RF hop acts as a bottleneck thereby leading to a floor in the curves for NBFSK at the demonstrated electrical SNRs of UOWC. Moreover, it can be observed for all Figures representing BER that the asymptotic expression derived in (32) of average BER at high SNR regimes i.e. above $15 \mathrm{~dB}$ on wards matches very tightly with its analytical expression proving the accuracy of the obtained asymptotic results.

\section{CONCLUDING REMARKS}

In this paper, we have derived exact closed-form expressions for the OP and BER via utilising Meijer's G functions that has not been exhibited in the open literature for a dual-hop E2E systems i.e. MIMO-RF for $S-R$ hop and UOWC for $R-D$ hop. Our derived analytical expressions for OP and BER present an efficient technique on the varying aspects of our system and channel parameters, namely the varying number of increasing antennas from $N_{t}=N_{r}=2,3,4$ or more from the source towards relay and the involvement of underwater detection techniques of $r=1$ for heterodyne detection and $r=2$ for IM/DD technique representing the water turbulence severity of the UOWC link, among many others. OP and BER along with their respective asymptotes applicable in high SNR regime for varying bubble levels (BL) (L/min) for different test cases, temperature gradients $\left({ }^{\circ} \mathrm{C}\right)$, fresh and saline waters, and binary modulation techniques have been accurately validated for the E2E system proposed and presented in this paper.

\section{REFERENCES}

[1] E. Illi, F. El Bouanani, D. B. Da Costa, F. Ayoub, and U. S. Dias, "Dual-hop mixed RF-UOW communication system: A. PHY security analysis," IEEE Access, vol. 6, pp. 55345-55360, Sep. 2018.
[2] Z. Zeng, S. Fu, H. Zhang, Y. Dong, and J. Cheng, "A survey of underwater optical wireless communication," IEEE Commun. Surv. Tut., vol. 19, no. 1, pp. 204-238, Jan.-Mar. 2017.

[3] F. Campagnaro, F. Guerra, P. Casari, R. Diamant, and M. Zorzi, "Implementation of a multi-modal acoustic-optical underwater network protocol stack," in OCEANS 2016 - Shanghai, pp. 1-6, 2016.

[4] A. Celik, N. Saeed, B. Shihada, T. Y. Al-Naffouri, and M.-S. Alouini, "End-to-end performance analysis of underwater optical wireless relaying and routing techniques under location uncertainty," IEEE Trans. Wireless Commun., vol. 19, no. 2, pp. 1167-1181, Feb. 2020.

[5] M. Elamassie, F. Miramirkhani, and M. Uysal, "Performance characterization of underwater visible light communication," IEEE Trans. Commun., vol. 67, no. 1, pp. 543-552, Jan. 2019.

[6] M. Elamassie and M. Uysal, "Vertical underwater visible light communication links: Channel modeling and performance analysis," IEEE Trans. Wireless Commun., vol. 19, no. 10, pp. 6948-6959, Oct. 2020.

[7] M. V. Jamali et al., "Statistical distribution of intensity fluctuations for underwater wireless optical channels in the presence of air bubbles," in Proc. Iran Workshop Commun. Inform. Theory, 2016, pp. 1-6.

[8] M. V. Jamali et al., "Statistical studies of fading in underwater wireless optical channels in the presence of air bubble, temperature, and salinity random variations (long version)," IEEE Trans. Commun., vol. 66, pp. 4706-4723, Oct. 2018.

[9] E. Zedini, H. M. Oubei, A. Kammoun, M. Hamdi, B. S. Ooi, and M.-S. Alouini, "A new simple model for underwater wireless optical channels in the presence of air bubbles," in Proc. IEEE Glob. Commun. Conf., 2017, pp. 1-6.

[10] E. Zedini, H. M. Oubei, A. Kammoun, M. Hamdi, B. S. Ooi, and M.-S Alouini, "Unified statistical channel model for turbulence-induced fading in underwater wireless optical communication systems," IEEE Trans. Commun., vol. 67, no. 4, pp. 2893-2907, Apr. 2019.

[11] S. Kumar, S. Prince, J. V. Aravind, and S. K. G., "Analysis on the effect of salinity in underwater wireless optical communication," Mar. Georesources Geotechnol., vol. 38, no. 3, pp. 291-301, 2020.

[12] E. Zedini, A. Kammoun, H. Soury, M. Hamdi, and M.-S. Alouini, "Performance analysis of dual-hop underwater wireless optical communication systems over mixture exponential-generalized gamma turbulence channels," IEEE Trans. Commun., vol. 68, no. 9, pp. 5718-5731, Sep. 2020

[13] Z. Chen, Z. Chi, Y. Li, and B. Vucetic, "Error performance of maximalratio combining with transmit antenna selection in flat nakagami-m fading channels," IEEE Trans. Wireless Commun., vol. 8, no. 1, pp. 424-431, Jan. 2009.

[14] N. Yang, M. Elkashlan, and J. Yuan, "Dual-hop amplify-and-forward MIMO relaying with antenna selection in nakagami-m fading," in Proc. IEEE Glob. Telecommun. Conf. GLOBECOM 2010, 2010, pp. 1-6.

[15] P. L. Yeoh, M. Elkashlan, and I. B. Collings, "MIMO relaying: Distributed TAS/MRC in Nakagami- $m$ fading," IEEE Trans. Commun., vol. 59, no. 10, pp. 2678-2682, Oct. 2011.

[16] T. Gucluoglu and T. M. Duman, "Performance analysis of transmit and receive antenna selection over flat fading channels," IEEE Trans. Wireless Commun., vol. 7, no. 8, pp. 3056-3065, Aug. 2008.

[17] A. Yilmaz and O. Kucur, "Performance of transmit antenna selection and maximal-ratio combining in dual hop amplify-and-forward relay network over nakagami-m fading channels," Wireless Pers. Commun., vol. 67, pp. 485-503, Dec. 2011.

[18] Z. Vali, A. Gholami, Z. Ghassemlooy, M. Omoomi, and D. G. Michelson, "Experimental study of the turbulence effect on underwater optical wireless communications," Appl. Opt., vol. 57, pp. 8314-8319, Oct. 2018.

[19] Y. Lou, R. Sun, J. Cheng, S. Liu, F. Zhou, and G. Qiao, "Physicallayer security for two-hop air-to-underwater communication systems with fixed-gain amplify-and-forward relaying," CoRR, vol. abs/2009.09550, Sep. 2020. [Online]. Available: https://arxiv.org/abs/2009.09550

[20] F. D. Ledezma et al., "A Market Survey of Offshore Underwater Robotic Inspection Technologies for the Oil and Gas Industry," in SPE Saudi Arabia Sect. Аnпи. Tech. Symp. Exhib., Apr. 21-23, 2015, pp. SPE-177989-MS.

[21] H. Kaushal and G. Kaddoum, "Underwater optical wireless communication," IEEE Access, vol. 4, pp. 1518-1547, Apr. 2016.

[22] B. Pranitha and L. Anjaneyulu, "Analysis of underwater acoustic communication system using equalization technique for ISI reduction," Procedia Comput. Sci., vol. 167, pp. 1128-1138, 2020.

[23] A. C. Boucouvalas, K. P. Peppas, K. Yiannopoulos, and Z. Ghassemlooy, "Underwater optical wireless communications with optical amplification and spatial diversity," IEEE Photon. Technol. Lett., vol. 28, no. 22, pp. 2613-2616, Nov. 2016. 
[24] H. M. Oubei et al., "Simple statistical channel model for weak temperatureinduced turbulence in underwater wireless optical communication systems," Opt. Lett., vol. 42, pp. 2455-2458, Jul. 2017.

[25] S. Anees and R. Deka, "On the performance of df based dual-hop mixed RF/UWOC system,” in Proc. IEEE 89th Veh. Technol. Conf., 2019, pp. 1-5.

[26] P. Ramavath, U. Acharya, and P. K., "Co-operative RF-UWOC link performance over hyperbolic tangent log-normal distribution channel with pointing errors," Opt. Commun., vol. 469, Mar. 2020, pp. 1-7, Art. no. 125774.

[27] Y. Lou, R. Sun, J. Cheng, D. Nie, and G. Qiao, "Secrecy outage analysis of two-hop decode-and-forward mixed RF/UWOC systems," IEEE Commun. Lett., to be published, doi: 10.1109/LCOMM.2021.3058988.

[28] H. Lei, Y. Zhang, K.-H. Park, I. S. Ansari, G. Pan, and M.-S. Alouini, "Performance analysis of dual-hop RF-UWOC systems," IEEE Photon. J., vol. 12, no. 2, pp. 1-15, Apr. 2020.

[29] N. Yang, P. L. Yeoh, M. Elkashlan, R. Schober, and I. B. Collings, "Transmit antenna selection for security enhancement in MIMO wiretap channels," IEEE Trans. Commun., vol. 61, no. 1, pp. 144-154, Jan. 2013.

[30] M. H. Protter and C. B. Morrey in A First Course in Real Analysis. New York, NY: Springer, 2nd ed., 2012.

[31] D. Zwillinger and V. Moll, in Table of Integrals, Series, and Products 8th ed., Boston: Academic Press, 8th ed., 2014.

[32] S. Choi and Y.-C. Ko, "Performance of selection MIMO systems with generalized selection criterion over Nakagami- $m$ fading channels," IEICE Trans. Commun.., vol. E89-B, no. 12, pp. 3467-3470, Dec. 2006.

[33] R. Beals and J. Szmigielski, "Meijer G-functions: A gentle introduction," Notices Amer. Math. Soc., vol. 60, pp. 866-872, Aug. 2013.

[34] I. S. Ansari, S. Al-Ahmadi, F. Yilmaz, M.-S. Alouini, and H. Yanikomeroglu, "A new formula for the BER of binary modulations with dual-branch selection over generalized-k composite fading channels," IEEE Trans. Commun., vol. 59, no. 10, pp. 2654-2658, Oct. 2011.

[35] K. S. Kolbig, I. S. Gradshteyn, I. M. Ryzhik, A. Jeffrey, and I. Scripta Technica, Table Integrals, Series, Products., 1995.

[36] V. S. Adamchik and O. I. Marichev, "The algorithm for calculating integrals of hypergeometric type functions and its realization in REDUCE system," in Proc. Int. Symp. Symbolic Algebr. Computation, ACM Press, 1990, pp. 212-224.

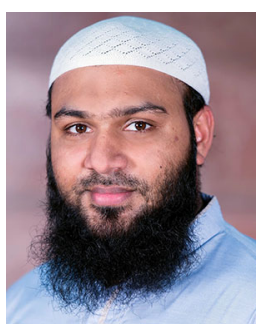

Imran Shafique Ansari (Member, IEEE) received the B.Sc. degree in computer engineering (with First Hons.) from the King Fahd University of Petroleum and Minerals (KFUPM) in 2009, and the M.Sc. and Ph.D. degrees from the King Abdullah University of Science and Technology (KAUST), in 2010 and 2015, respectively. Since August 2018, he has been a Lecturer (Assistant Professor) with University of Glasgow, Glasgow, U.K. Prior to this, from November 2017 to July 2018, he was a Lecturer (Assistant Professor) with Global College of Engineering and Technology (GCET) (affiliated with University of the West of England (UWE), Bristol, U.K.). From April 2015 to November 2017, he was a Postdoctoral Research Associate (PRA) with Texas A\&M University with Qatar (TAMUQ). From May 2009 to August 2009, he was a Visiting Scholar with Michigan State University (MSU), East Lansing, MI, USA, and from June 2010 to August 2010, he was a Research Intern with Carleton University, Ottawa, ON, Canada.

He has been affiliated with IEEE since 2007 and was in various capacities. He is the IEEE Nominations and Appointments (N\&A) Committee since 2020-2021 and IEEE Communication Society Young Professionals (ComSoc YP) Board since April 2016. He is part of the IEEE 5G Tech Focus Publications Editorial Board since February 2017. He is an active reviewer for various IEEE TRANSACTIONS and various other journals. He was a TPC for various IEEE conferences. Dr. Ansari has authored or coauthored more than 100 journal and conference publications. He has Co-Organized the GRASNET'2016, 2017, 2018 workshops in conjunction with IEEE WCNC'2016, 2017 and IEEE Globecom 2018. His research interests include free-space optics (FSO), underwater communications, physical layer secrecy issues, full duplex systems, and secure D2D applications for $5 \mathrm{G}+$ systems, among others. He was the recipient of appreciation for an Exemplary Reviewer for IEEE Transaction on Communications (TCOM) in 2018 and 2016, the recipient of appreciation for an Exemplary Reviewer for IEEE Wireless Communications Letters (WCL) in 2017 and 2014, recipient of TAMUQ ECEN Research Excellence Award 2016, 2017, recipient of Postdoctoral Research Award (PDRA) (first cycle) with Qatar national research foundation (QNRF) in 2014, recipient of KAUST academic excellence award (AEA) in 2014, and the recipient of IEEE Richard E. Merwin student scholarship award in July 2013.

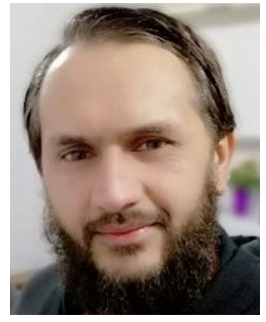

Latif Jan (Member, IEEE) completed the M.Sc. degree in the field of wireless communications from UET Peshawar. He is currently the Ph.D. Scholar with University of Engineering \& Technology (UET), Peshawar, Pakistan. He is currently a Lecturer with Iqra National University (INU) Peshawar. His research interests include the field of optical wireless communication systems, physical layer security and free space optics for MIMO and massive MIMO technologies.

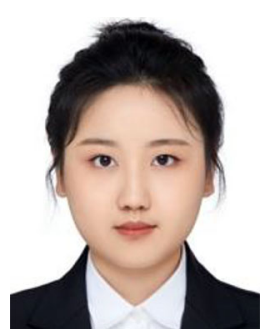

Yutong Tang received the B.E. degree (Hons.) from Glasgow College, University of Electronic Science and Technology of China, Chengdu, China, in 2021. She is currently working toward the Ph.D. degree with the University of Glasgow. Her current research interests include on the modeling, design, and performance analysis of wireless communication systems, with an emphasis on optical wireless communication systems on the basis of reconfigurable intelligent surfaces.

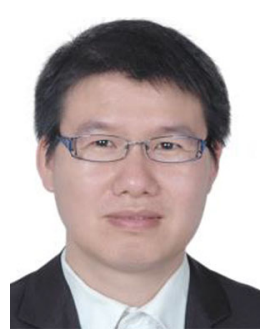

Liang Yang (Member, IEEE) was born in Hunan, China. He received the Ph.D. degree in electrical engineering from Sun Yat-sen University, Guangzhou, China, in 2006. From 2006 to 2013, he was a Faculty with Jinan University, Guangzhou, China. He joined the Guangdong University of Technology in 2013. He is currently a Professor with Hunan University, Changsha, China. His current research interests include the performance analysis of wireless communications systems.

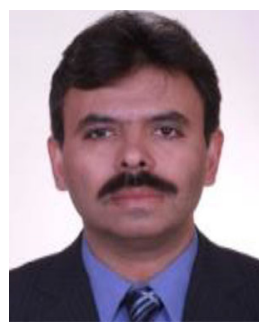

Mohammad Haseeb Zafar (Senior Member, IEEE) received the Ph.D. degree in electronic and electrical engineering (EEE) from the University of Strathclyde in 2009. He is currently a Professor with the Faculty of Electrical and Computer Engineering, the University of Engineering \& Technology, Peshawar, Pakistan. $\mathrm{He}$ is also a Professor with the Faculty of Computing and Information Technology, King Abdulaziz University, Jeddah, Saudi Arabia. His research interests include performance analysis of diverse computer and wireless communication networks and systems. He is lifetime member of Pakistan Engineering Council. 